\title{
NNIL-formulas revisited: universal models and finite model property
}

\author{
Julia Ilin*, Dick de Jongh*, and Fan Yang ${ }^{\dagger}$ \\ *Institute of Logic, Language and Computation, University of Amsterdam. P.O. \\ Box 94242, 1090 GE Amsterdam, The Netherlands, email: ilin.juli@gmail.com, \\ d.h.j.dejongh@uva.nl \\ ${ }^{\dagger}$ Department of Mathematics and Statistics, University of Helsinki, PL 68 (Pietari \\ Kalmin katu 5), 00014 University of Helsinki, Finland, email: \\ fan.yang.c@gmail.com
}

\begin{abstract}
NNIL-formulas, introduced by Visser in 1983-1984 in a study of $\Sigma_{1}$-subsitutions in Heyting Arithmetic, are intuitionistic propositional formulas that does not allow nesting of implication to the left. The first results about these formulas were obtained in a paper of 1995 by Visser et al. In particular, it was shown that NNIL-formulas are exactly the formulas preserved under taking submodels of Kripke models. Recently Bezhanishvili and de Jongh observed that NNIL-formulas are also reflected by color-preserving monotonic maps of Kripke models. In the present paper, we first show how this observation leads to the conclusion that NNIL-formulas are preserved by arbitrary substructures not necessarily satisfying the topo-subframe condition. Then we apply it to construct universal models for NNIL. It follows from the properties of these universal models that NNIL-formulas are also exactly the formulas that are reflected by color-preserving monotonic maps. By using the method developed in constructing the universal models, we give a new direct proof that the logics axiomatized by NNIL-axioms have the finite model property.
\end{abstract}

Keywords - Intuitionistic logic, universal model, finite model property, subframe formulas, monotonic maps.

\section{Introduction}

NNIL-formulas are formulas with no nesting of implications to the left. These formulas are very expressive but considerably easier to handle and 
less complex than the class of all formulas in the language of the intuitionistic propositional calculus IPC, as seen e.g., in the connection with infon logics [9] and in the fact the class of NNIL-formulas is locally finite, i.e., there are only finitely many non-equivalent NNIL-formulas in $n$ variables for every $n$. The study of these formulas was introduced by Visser in 19831984 when working on $\Sigma_{1}$-substitutions of propositional formulas in Heyting Arithmetic [27], an investigation that was continued in cooperation with de Jongh [12]. Research on the purely propositional properties of these formulas was undertaken in [28]. The main tool in that article was the use of subsimulations, a type of simulation that leaves the valuation of NNIL-formulas intact. It is shown that NNIL-formulas are (up to provable equivalence) exactly the ones that are preserved under taking submodels of Kripke models. In [3] and in [29] it was remarked that this implies that NNIL-formulas are also preserved under taking subframes. They axiomatize so-called subframe logics. Modal subframe logics were first introduced by Fine [13], and intermediate subframe logics were defined by Zakharyaschev [30] (see also [8, $\S 11.3]$ ), who also proved the finite model property of these logics.

It may be thought of as surprising that NNIL-formulas axiomatize subframe logics, because for example [2] used $[\wedge, \rightarrow]$-formulas (i.e., formulas that have $\wedge$ and $\rightarrow$ only as connectives) to axiomatize these logics and to prove their finite model property. The $[\wedge, \rightarrow]$-formulas, though forming a locally finite class as well, are very different in character from NNIL-formulas. To obtain NNIL-axiomatizations, in [3] (see also [6]), for each finite rooted frame $\mathfrak{F}$, a NNIL-formula is constructed from a model $\mathfrak{M}$ on that frame that fails on a descriptive frame $\mathfrak{G}$ iff $\mathfrak{F}$ is a p-morphic image of a subframe of $\mathfrak{G}$, as one calls it, a refutation criterion. Using ideas from [6] in this paper we show that monotonic maps can be used to describe the behavior of such formulas if the maps satisfy an additional condition: color-consistency. The formulas fail on a descriptive frame $\mathfrak{G}$ iff the unraveling of $\mathfrak{M}$ to a tree can be mapped into $\mathfrak{G}$ by a color-consistent monotonic function. This is shown to lead to the conclusion that the frame classes of intermediate subframe logics, because they are axiomatized by NNIL-formulas, are closed under subframes in general, even of they do not satisfy the topo-subframe condition (Corollary 3.8). This means that the class of intermediate subframe logics is at least in some ways essentially less complicated than the class of modal subframe logics. This is certainly connected to the fact shown in [28] that NNIL-formulas are the ones of which the standard correspondent as a first order formula is equivalent to a universal one, even if it may not yet be quite clear how.

We will further exploit the obtained refutation criterion via color-consis- 
tent monotonic maps in the present paper in two ways. A first central result is a full description of the $n$-universal model $\mathcal{T}(n)$ for NNIL-formulas. This will complete the work started in [29]. In fact it turns out that $\mathcal{T}(n)$ is also the universal model for monotonically reflective formulas (MR-formulas), the class of formulas whose validity is reflected (or backwards preserved) by monotonic maps. NNIL-formulas are easily seen to be MR-formulas, and it follows essentially from [28, that NNIL-formulas are also exactly the ones reflected by monotonic maps. We will give an alternative proof of this result as a corollary of our universal model construction. The facts that logics axiomatized by NNIL-formulas have the finite model property and are canonical will also be a simple consequence of our investigations into color-consistent monotonic maps. As logics axiomatized by NNIL-formulas correspond to subframe logics, these results are not new. What is new is, as stated above, that the frames of intermediate subframe logics defined by NNIL-formulas, hence all intermediate subframe logics, are closed under arbitrary substructures not necessarily satisfying the topo-subframe condition.

The paper is organized as follows: Section 2 contains the preliminaries of the paper. In Section 3 we prove the refutation criterion for NNIL-formulas via (color-consistent) monotonic maps. Section 4 constructs universal models for NNIL-formulas, and Section 5 proves the finite model property for logics axiomatized by NNIL-formulas. We conclude in Section 6 by mentioning some open problems.

This article is largely based on [17. The proof of the finite model property (Theorem [5.8) has already appeared in [18, a paper dedicated to Albert Visser.

\section{Preliminaries}

In this section, we recall briefly the most relevant terminologies and notations of this paper; for a more extensive treatment we refer the reader to [6] and [3, 8, 10]. We fix a set Prop of propositional variables $p$. Formulas of intuitionistic propositional logic are defined by the grammar:

$$
\varphi:=\perp|p| \varphi \wedge \varphi|\varphi \vee \varphi| \varphi \rightarrow \varphi
$$

As usual, we write $\neg \varphi$ for $\varphi \rightarrow \perp$. We also adopt the usual connective precedence that $\neg$ has higher precedence than $\wedge$ and $\vee$, which have precedence than $\rightarrow$. For instance $p \vee q \rightarrow \neg r \wedge s$ is read as $(p \vee q) \rightarrow((\neg r) \wedge s)$. We consider the usual intuitionistic propositional calculus IPC, and write $\vdash_{\text {IPC }} \varphi$ 
or simply $\vdash \varphi$ if $\varphi$ is a theorem of IPC. An intermediate logic $L$ is a set of formulas containing IPC closed under modus ponens and substitution.

We have the usual Kripke semantics with intuitionistic (Kripke) frames $\mathfrak{F}=(W, R)$ and models $\mathfrak{M}=(\mathfrak{F}, V)$, where $W$ is a nonempty set of worlds (also called points or nodes), $R$ is a partial order on $W$, and $V$ is a persistent valuation (i.e., $w \in V(p)$ and $w R u$ imply $u \in V(p)$ ). For any world $w$, define $R(w)=\{u \in W \mid w R u\}$ and $R^{-1}(w)=\{u \in W \mid u R w\}$. A set $U \subseteq W$ is said to be upward closed (or an upset) if $w \in U$ and $w R u$ imply $u \in U$. Denote by $U p(W)$ the set of all upsets of $W$. An intuitionistic general frame $\mathfrak{F}=(W, R, \mathcal{P})$ is an intuitionistic $\operatorname{Kripke}$ frame $(W, R)$ equipped with a set $\mathcal{P}$ of upsets containing $\emptyset$ and $W$, and closed under $\cup, \cap$ and the binary operator $\rightarrow$ on $\wp(W)$ defined as

$$
U_{0} \rightarrow U_{1}:=\left\{w \in W \mid \forall v: w R v \text { and } v \in U_{0} \text { imply } v \in U_{1}\right\} .
$$

An intuitionistic general frame $\mathfrak{F}$ is called refined if $\neg w R v$ implies that there is $U \in \mathcal{P}$ such that $w \in U$ and $v \notin U ; \mathfrak{F}$ is called compact if for every $\mathcal{X} \subseteq \mathcal{P} \cup\{W \backslash U \mid U \in \mathcal{P}\}$, if $\mathcal{X}$ has the finite intersection property (i.e., $\bigcap \mathcal{X}_{0} \neq \emptyset$ for every finite subset $\left.\mathcal{X}_{0} \subseteq \mathcal{X}\right)$, then $\bigcap \mathcal{X} \neq \emptyset$. An intuitionistic descriptive frame $\mathfrak{F}=(W, R, \mathcal{P})$ is an intuitionistic general frame that is both refined and compact. In particular, a Kripke frame $\mathfrak{F}$ can be seen as a general frame $(\mathfrak{F}, \mathcal{P})$ with $\mathcal{P}=U p(W)$, which is clearly refined. A finite Kripke frame is also compact and thus descriptive. A descriptive model $\mathfrak{M}=(\mathfrak{F}, V)$ is a descriptive frame $\mathfrak{F}$ with a persistent valuation satisfying in addition $V(p) \in \mathcal{P}$ for all $p \in$ Prop. Formulas in the language of IPC are evaluated at a world $w$ in a (Kripke or descriptive) model $\mathfrak{M}$ recursively as follows:

$\mathfrak{M}, w \not \models \perp \quad$ always;

$\mathfrak{M}, w \models p \quad$ iff $\quad w \in V(p)$;

$\mathfrak{M}, w \models \varphi \wedge \psi \quad$ iff $\quad \mathfrak{M}, w \models \varphi$ and $\mathfrak{M}, w \models \psi$;

$\mathfrak{M}, w \models \varphi \vee \psi \quad$ iff $\quad \mathfrak{M}, w \models \varphi$ or $\mathfrak{M}, w \models \psi$;

$\mathfrak{M}, w \models \varphi \rightarrow \psi \quad$ iff $\quad$ for every $u$ with $w R u, \mathfrak{M}, u \models \varphi$ implies $\mathfrak{M}, u \models \psi$.

Define $V(\varphi)=\{w \in W \mid \mathfrak{M}, w \models \varphi\}$, and write $\mathfrak{M} \models \varphi$ if $V(\varphi)=W$. For a (descriptive) frame $\mathfrak{F}$ we write $\mathfrak{F} \models \varphi$ if $\mathfrak{M} \models \varphi$ for every model $\mathfrak{M}$ on $\mathfrak{F}$.

In this paper, we often consider IPC with respect to $n$ fixed propositional variables $p_{1}, \ldots, p_{n}$. By an $n$-formula we mean a formula whose propositional variables are among the $n$ fixed ones only. Similarly, an $n$-model is a model $(\mathfrak{F}, V)$ with the valuation $V$ restricted to the set consisting of the $n$ fixed 
propositional variables. We define the color of a point $w$ in an $n$-model, denoted $\operatorname{col}(w)$, as the sequence $i_{1} \ldots i_{n}$ with $i_{k}=1$ if $p_{i}$ is true in $w$, and 0 otherwise. We write $i_{1} \ldots i_{n} \leq j_{1} \ldots j_{n}$ iff $i_{k} \leq j_{k}$ for each $k=1, \ldots, n$, and $i_{1} \ldots i_{n}<j_{1} \ldots j_{n}$ if $i_{1} \ldots i_{n} \leq j_{1} \ldots j_{n}$ and $i_{1} \ldots i_{n} \neq j_{1} \ldots j_{n}$.

A (Kripke or descriptive) frame $\mathfrak{F}^{\prime}=\left(W^{\prime}, R^{\prime}, \mathcal{P}^{\prime}\right)$ is said to be a subframe of a (Kripke or descriptive) frame $\mathfrak{F}=(W, R, \mathcal{P})$ iff $W^{\prime} \subseteq W, R^{\prime}=R \uparrow W^{\prime}$, where in the case of a descriptive frame an additional topo-subframe condition needs to be satisfied, namely $W^{\prime} \backslash U \in \mathcal{P}^{\prime}$ implies $W \backslash R^{-1}(U) \in \mathcal{P}$ for all $U \subseteq W^{\prime}$ (see e.g., [6] for detailed discussion). We will also study subframes of descriptive frames which need not satisfy the topo-subframe condition. We call these substructures. Similarly, a model $\mathfrak{M}^{\prime}=\left(\mathfrak{F}^{\prime}, V^{\prime}\right)$ is a submodel of $\mathfrak{M}=(\mathfrak{F}, V)$ iff $\mathfrak{F}^{\prime}$ is a subframe of $\mathfrak{F}$ and $V^{\prime}(p)=V(p) \cap W$ for each $p$. We write $\mathfrak{M}_{w}$ for the submodel of $\mathfrak{M}$ generated by $w$, that is, the submodel with $R(w)$ as the domain; similarly for generated subframes $\mathfrak{F}_{w}$. It is easy to see that $\mathfrak{M}_{w} \models \varphi$ iff $\mathfrak{M}, w \models \varphi$ for any formula $\varphi$. If $\mathfrak{M}=\mathfrak{M}_{r}$ for some node $r$ (called the root), then $\mathfrak{M}$ is said to be rooted; similarly for rooted frames. Most frames and models we consider in this paper will be rooted.

If $w R u$, then we call $u$ a successor of $w$. If in addition $w \neq u$, then $u$ is called a proper successor of $w$, in symbols $w R^{+} u$. If $w R^{+} u$ and, for no $v$, $w R^{+} v R u, u$ is called an immediate successor of $w$. Points $w$ with no proper successor are called maximal, that is, $w R u$ implies $w=u$. The depth of a point $w$ in a finite model $\mathfrak{M}$ is defined as the maximal length $k$ of a chain $w=w_{1} R^{+} \ldots R^{+} w_{k}$ in $\mathfrak{M}$, in particular, the depth of a maximal point is 1 . The depth of a finite model is the maximal depth of the points in the model.

A model $(W, R, V)$ is called tree-like (or simply a tree) if for all $w \in W$, $R^{-1}(w)$ is finite and linearly ordered; similarly for tree-like frames. Recall that the standard unraveling of a rooted (infinite) model $\mathfrak{M}=(W, R, V)$ with root $r$ is a tree-like model $\mathfrak{M}_{\mathrm{t}}=\left(W_{\mathrm{t}}, R_{\mathrm{t}}, V_{\mathrm{t}}\right)$ defined as

- $W_{\mathrm{t}}=\left\{\left\langle r, w_{1}, \ldots, w_{k}\right\rangle \mid r R w_{1} R \ldots R w_{k}\right\}$,

- $\sigma R_{\mathrm{t}} \tau$ iff $\sigma$ is an initial segment of $\tau$,

- $\left\langle r, w_{1}, \ldots, w_{k}\right\rangle \in V_{\mathrm{t}}(p)$ iff $w_{k} \in V(p)$.

For finite rooted models $\mathfrak{M}$, the unraveling $\mathfrak{M}_{\mathrm{t}}$ is defined the same way except that $W_{\mathrm{t}}$ is defined using immediate successorship instead of successorship $R$. We identify the root $\langle r\rangle$ of the unraveled model $\mathfrak{M}_{\mathrm{t}}$ with the root $r$ of the original model $\mathfrak{M}$, and write simply $r$ for $\langle r\rangle$. 
One central notion of our revisit to NNIL-formulas is the notion of monotonic map. A monotonic map between two frames $\mathfrak{G}=(W, R)$ and $\mathfrak{F}=$ $\left(W^{\prime}, R^{\prime}\right)$ is a function $f: W \rightarrow W^{\prime}$ that preserves order, i.e.,

(order preservation) $w R u$ implies $f(w) R^{\prime} f(u)$.

A color-preserving monotonic map $f: \mathfrak{N} \rightarrow \mathfrak{M}$, denoted as $\mathfrak{M} \leq_{f} \mathfrak{N}$, is a monotonic map between the two underlying frames that also preserves colors, i.e.,

(color preservation) $\operatorname{col}(f(w))=\operatorname{col}(w)$.

We write $\mathfrak{M} \leq \mathfrak{N}$ if there exists some color-preserving monotonic map $f$ such that $\mathfrak{M} \leq_{f} \mathfrak{N}$. Note that functional subsimulations, which played an important role in the previous study [28] of NNIL-formulas, are, in fact, colorpreserving monotonic maps. Let us also recall that the familiar p-morphisms are color-preserving monotonic maps $f$ between two models that also satisfy:

(back condition) $f(w) R^{\prime} u^{\prime}$ implies $\exists u \in W^{\prime}\left(w R u\right.$ and $\left.f(u)=u^{\prime}\right)$.

As an example, the natural map $\alpha$ between an unraveled model $\mathfrak{M}_{\mathrm{t}}$ and the original model $\mathfrak{M}$, defined as $\alpha\left(\left\langle r, w_{1}, \ldots, w_{k}\right\rangle\right)=w_{k}$, is a surjective pmorphism. It is easy to see that the truth of a formula $\varphi$ is invariant under p-morphisms $f$ between two models $\mathfrak{N}$ and $\mathfrak{M}$, i.e.,

$$
\mathfrak{M}, f(w) \models \varphi \Longleftrightarrow \mathfrak{N}, w \models \varphi .
$$

Let us now recall from [28, 29] some basic facts about NNIL-formulas, which are formulas in the language of IPC with no nesting of implications to the left. For example, $p \vee q \rightarrow(r \rightarrow s)$ and $p \rightarrow(q \rightarrow \perp) \vee(r \rightarrow s)$ are NNIL-formulas, whereas $(p \rightarrow \perp) \rightarrow \perp$ and $(p \rightarrow q) \vee r \rightarrow p$ are not. NNILformulas form a locally finite class of formulas, that is, for every natural number $n$, there are only finitely many non-equivalent NNIL-formulas in $n$ propositional variables. For short, we may say somewhat improperly that NNIL-formulas are locally finite. Since conjunctions and disjunctions in front of implication can be eliminated using the equivalences

$\vdash(\varphi \wedge \psi \rightarrow \chi) \leftrightarrow(\varphi \rightarrow(\psi \rightarrow \chi)$ and $\vdash(\varphi \vee \psi \rightarrow \chi) \leftrightarrow((\varphi \rightarrow \psi) \wedge(\varphi \rightarrow \chi))$,

every NNIL-formula can be brought into an equivalent NNIL-formula in the following normal form:

Definition 2.1. NNIL-formulas in normal form are defined by the grammar:

$$
\varphi:=\perp|p| \varphi \wedge \varphi|\varphi \vee \varphi| p \rightarrow \varphi
$$


The approach of this paper is based on the observation made in [6] that NNIL-formulas are reflected (or backwards preserved) by color-preserving monotonic maps. We recall this fact in detail as follows.

Lemma 2.2. [6] Let $\varphi$ be a NNIL-formula. For any two models $\mathfrak{N}=$ $(W, R, V)$ and $\mathfrak{M}=\left(W^{\prime}, R^{\prime}, V^{\prime}\right)$ such that $\mathfrak{M} \leq_{f} \mathfrak{N}$ for some color-preserving monotonic map $f: \mathfrak{N} \rightarrow \mathfrak{M}$, we have that for any $w \in W$,

$$
\mathfrak{M}, f(w) \models \varphi \Longrightarrow \mathfrak{N}, w \models \varphi .
$$

In particular, if $\mathfrak{M} \leq \mathfrak{N}$ and $\mathfrak{M} \models \varphi$, then $\mathfrak{N} \models \varphi$.

Proof. The proof is a routine induction on $\varphi$. Assume $\varphi$ to be in normal form. Only the case $\varphi=p \rightarrow \psi$ is non-trivial. Suppose $\mathfrak{M}, f(w) \models p \rightarrow \psi$ and $\mathfrak{N}, u \models p$ for some $u$ with $w R u$. Since $f$ is monotonic and colorpreserving, $f(w) R^{\prime} f(u)$ and $\mathfrak{M}, f(u) \models p$, thus $\mathfrak{M}, f(u) \models \psi$. By the induction hypothesis, we obtain $\mathfrak{N}, u \models \psi$, as required.

The above lemma also gives rise to the class MR (short for monotonically reflective) of formulas that are reflected by color-preserving monotonic maps 1 Obviously we have NNIL $\subseteq$ MR.

The identity map from a submodel $\mathfrak{N}$ of $\mathfrak{M}$ to $\mathfrak{M}$ itself is obviously a color-preserving monotonic map. Consequently, NNIL-formulas $\varphi$ are preserved under submodels, that is, $\mathfrak{M} \models \varphi$ implies $\mathfrak{N} \models \varphi$. It was shown in 28 that the converse holds as well, namely, every formula preserved under taking submodels is (equivalent to) a NNIL-formula. From this it also follows that every MR-formula (being preserved under taking submodels) is equivalent to some NNIL-formula, and thus NNIL $=$ MR. In this paper (in Corollary 4.11) we provide a direct alternative proof of these results by means of monotonic maps.

\section{NNIL-subframe formulas and monotonic maps on trees}

In this section we present a refutation criterion for NNIL-subframe formulas via monotonic maps. NNIL-subframe formulas were first introduced in [3, $\S 3.3]$ as formulas axiomatizing subframe logics in NNIL-form. They were inspired by the Jankov-de Jongh formulas, in fact they were introduced together in [3]. In the universal model of IPC (see Definition 4.2) the Jankov-de

\footnotetext{
${ }^{1}$ The class MR was called SR (short for stably reflective) in [17.
} 
Jongh formulas characterize point generated upsets. Their validity can thus be translated into a tangible semantic condition which leads to a refutation criterion (known as the Jankov-de Jongh Theorem). In the case of the NNIL-subframe formulas the refutation condition (via p-morphisms) comes immediately and was used in [3] to show that these formulas axiomatize all subframe logics (i.e., logics whose class of frames is closed under subframes).

In [3] (see also [6]) the NNIL-subframe formulas were introduced as certain NNIL-formulas $\beta(\mathfrak{F})$ constructed inductively out of arbitrary finite rooted frames $\mathfrak{F}$. Such constructions make sense for arbitrary finite models as well. We now define NNIL-subframe formulas $\beta(\mathfrak{N})$ (in $n$ variables) with respect to arbitrary finite $n$-models $\mathfrak{N}$ in the same manner. This slight difference in the definition will enable us to prove a simpler refutation criterion for NNIL-subframe formulas via monotonic maps, which will be important for the remaining sections of the paper.

Definition 3.1. Let $\mathfrak{N}=(W, R, V)$ be a finite $n$-model. For every $w \in W$, we define a NNIL-formula $\beta(w)$ by induction on the depth of $w$ as follows:

- If $w$ is a maximal point of $\mathfrak{N}$, define

$$
\beta(w):=\bigwedge \operatorname{prop}(w) \rightarrow \bigvee \text { notprop }(w)
$$

where

$$
\begin{gathered}
\operatorname{prop}(w):=\left\{p_{i} \mid \mathfrak{N}, w \models p_{i}, 1 \leq i \leq n\right\} \\
\text { and } \operatorname{notprop}(w):=\left\{p_{i} \mid \mathfrak{N}, w \not \models p_{i}, 1 \leq i \leq n\right\} .
\end{gathered}
$$

- If $w$ is not maximal, and $w_{1}, \ldots, w_{k}$ are all of its immediate successors with $\beta\left(w_{i}\right)$ already defined for every $w_{i}$, then define

$$
\beta(w):=\bigwedge \operatorname{prop}(w) \rightarrow \bigvee \text { notprop }(w) \vee \bigvee_{i=1}^{k} \beta\left(w_{i}\right)
$$

If $\mathfrak{N}$ is rooted with root $r$, we define $\beta(\mathfrak{N})=\beta(r)$.

Note that the formula $\beta(w)$ is also parameterized with the natural number $n$, which is given by the $n$-model $\mathfrak{N}$ that the node $w$ is taken from.

Lemma 3.2. For any finite $n$-model $\mathfrak{N}$, we have $\mathfrak{N}, w \not \models \beta(w)$.

Proof. We prove the lemma by induction on $d(w)$. If $d(w)=1$, clearly,

$$
\mathfrak{N}, w \models \bigwedge \operatorname{prop}(w) \text { and } \mathfrak{N}, w \not \models \bigvee \operatorname{notprop}(w),
$$


which give $\mathfrak{N}, w \not \models \beta(w)$.

Suppose $d(w)>1$ and the lemma holds for all nodes with depth less than $d(w)$. Assume that $w_{1}, \ldots, w_{k}$ are immediate successors of $w$. By induction hypothesis, we have $\mathfrak{N}, w_{i} \not \models \beta\left(w_{i}\right)$ for all $1 \leq i \leq k$. Thus, we obtain $\mathfrak{N}, w \not \models \bigvee_{i=1}^{k} \beta\left(w_{i}\right)$ by persistency. Since (2) also holds for $w$, we conclude $\mathfrak{N}, w \not \models \beta(w)$.

We now prove our new refutation criterion for the NNIL-subframe formulas $\beta(\mathfrak{N})$ via monotonic maps. In this criterion and also in other discussions in the sequel, we will consider the unraveled tree-like models $\mathfrak{N}_{\mathrm{t}}$ instead of the finite rooted $n$-models $\mathfrak{N}$ themselves. For reasons that will become apparent in the detailed proofs, it is in fact technically crucial to do so. This subtlety was already apparent in the previous study of universal models for NNIL-formulas in [29]. Since any node $w$ in the finite $n$-model $\mathfrak{N}$ with root $r$ and the corresponding node $\langle r, \ldots, w\rangle$ in $\mathfrak{N}_{\mathrm{t}}$ have the same color and essentially the same set of immediate successors, one can show by induction that the two formulas $\beta(\mathfrak{N})$ and $\beta\left(\mathfrak{N}_{\mathbf{t}}\right)$ are actually identical. We will thus not distinguish between the two formulas $\beta(\mathfrak{N})$ and $\beta\left(\mathfrak{N}_{\mathfrak{t}}\right)$.

Theorem 3.3. Let $\mathfrak{M}$ be an $n$-model and $\mathfrak{N}$ a finite rooted $n$-model. Then, $\mathfrak{M} \not \models \beta(\mathfrak{N})$ iff $\mathfrak{M} \leq \mathfrak{N}_{\mathrm{t}}$.

In particular, for any (Kripke or descriptive) frame $\mathfrak{F}$, we have that $\mathfrak{F} \not \models \beta(\mathfrak{N})$ iff $\mathfrak{M} \leq \mathfrak{N}_{\mathrm{t}}$ for some model $\mathfrak{M}$ on $\mathfrak{F}$.

Proof. Suppose first that $\mathfrak{M} \leq \mathfrak{N}_{\mathrm{t}}$ and $r$ is the root of $\mathfrak{N}_{\mathrm{t}}$. By Lemma 3.2, we have $\mathfrak{N}_{\mathrm{t}}, r \not \neq \beta(r)$. Since $\beta(r) \in \mathrm{NNIL}$, we obtain by Lemma 2.2 that $\mathfrak{M} \not \models \beta(r)$, i.e., $\mathfrak{M} \not \forall \beta(\mathfrak{N})$.

Conversely, assuming $\mathfrak{M} \not \beta \beta(\mathfrak{N})$ we define a color-preserving monotonic map $f: \mathfrak{N}_{\mathrm{t}} \rightarrow \mathfrak{M}$ by defining the value $f(u)$ by induction on the depth of $u$ in $\mathfrak{N}_{\mathrm{t}}$. We will guarantee that $f$ is monotonic and color-preserving by guaranteeing the following: (i) $f(u)$ has the color of $u$. (ii) For the unique immediate predecessor $w$ of $u, f(u)$ is a successor of $f(w)$. (iii) For every immediate successor $u_{i}$ of $u, \mathfrak{M}, f(u) \not \models \beta\left(u_{i}\right)$.

We first define $f(r)$. Since $\mathfrak{M} \not \models \beta(r)$, there exists a node $x$ in $\mathfrak{M}$ such that

$$
\mathfrak{M}, x \models \bigwedge p \operatorname{prop}(r), \mathfrak{M}, x \not \models \bigvee \operatorname{notprop}(r) \text { and } \mathfrak{M}, x \not \models \beta\left(r_{i}\right)
$$

for each immediate successor $r_{i}$ of $r$ (if any). We define $f(r)=x$. Clearly (3) implies that $\operatorname{col}(f(r))=\operatorname{col}(x)=\operatorname{col}(r)$ and $\mathfrak{M}, f(r) \not \models \beta\left(r_{i}\right)$.

Next, we define $f(u)$ for $u$ properly above $r$. Let $w$ the unique immediate predecessor of $u$ in the unraveled tree-like model $\mathfrak{N}_{\mathrm{t}}$, and suppose that $f(w)$ 
has already been defined. Similar to the above, since we have guaranteed that $\mathfrak{M}, f(w) \forall \forall \beta(u)$, there is a successor $x$ of $f(w)$ for which the corresponding clause (3) holds for $x$ and $u$. Define $f(u)=x$. By definition $f(u)$ is a successor of $f(w)$. Again, (3) implies that $\operatorname{col}(f(u))=\operatorname{col}(x)=\operatorname{col}(u)$ and $\mathfrak{M}, f(u) \not \models \beta\left(u_{i}\right)$ for each immediate successor $u_{i}$ of $u$.

Let B denote the collection of all NNIL-subframe formulas of finite models as defined in Definition 3.1, i.e.

$$
\mathrm{B}=\{\beta(w) \mid w \text { is a node in some finite } n \text {-model for some } n\} .
$$

Obviously, B is included in the class of NNIL-formulas. As mentioned already, N. Bezhanishvili [3] gave the refutation criterion for formulas in B via p-morphisms, which are color-preserving monotonic maps with extra conditions. In this sense our Theorem 3.3 is simpler than the corresponding one in [3]. On the basis of the refutation criterion N. Bezhanishvili was able to prove that the formulas in $\mathrm{B}$ are sufficient to axiomatize the intermediate subframe logics and hence

Theorem 3.4 ([3], Cor. 3.4.16). All intermediate subframe logics are axiomatized by NNIL-formulas.

Recall that by Lemma 2.2 all NNIL-formulas are in MR. As another consequence of Theorem 3.3. the three formula classes B, NNIL and MR distinguish the same finite pointed models in the sense of the following definition. This result for NNIL- and MR-formulas follows also already from 28], by a different argument.

Definition 3.5. Let $\Phi$ be a class of formulas. Two pairs $(\mathfrak{M}, w)$ and $(\mathfrak{N}, u)$ of models with nodes in the corresponding domains are said to be $\Phi$-equivalent, written $(\mathfrak{M}, w) \simeq_{\Phi}(\mathfrak{N}, u)$, if for each $\varphi \in \Phi$,

$$
\mathfrak{M}, w \models \varphi \Longleftrightarrow \mathfrak{N}, u \models \varphi .
$$

Similarly, we write $\mathfrak{M} \simeq_{\Phi} \mathfrak{N}$ if for each $\varphi \in \Phi$,

$$
\mathfrak{M} \models \varphi \Longleftrightarrow \mathfrak{N} \models \varphi .
$$

Proposition 3.6. Let $\mathfrak{M}$ and $\mathfrak{N}$ be finite models with nodes $w$ and $u$ in the corresponding domains, respectively. The following are equivalent:

(i) $(\mathfrak{M}, w) \simeq_{\mathrm{B}}(\mathfrak{N}, u)$.

(ii) $(\mathfrak{M}, w) \simeq_{\mathrm{NNIL}}(\mathfrak{N}, u)$. 
(iii) $(\mathfrak{M}, w) \simeq_{\mathrm{MR}}(\mathfrak{N}, u)$.

Proof. The implications (iii) $\Rightarrow$ (ii) $\Rightarrow$ (i) are obvious since $\mathrm{B} \subseteq$ NNIL $\subseteq$ MR. We show that (i) implies (iii). Assume that $(\mathfrak{M}, w) \simeq_{\mathrm{B}}(\mathfrak{N}, u)$. For any $\varphi \in \mathrm{MR}$, we only show the direction that $\mathfrak{M}, w \models \varphi$ implies $\mathfrak{N}, u \models \varphi$; the other direction is symmetric. Assume that $\varphi$ is an $n$-formula and view $\mathfrak{N}$ and $\mathfrak{M}$ as $n$-models by restricting the valuations to the variables of $\varphi$. By Lemma 3.2, $\mathfrak{N}, u \not \models \beta(u)$, which by B-equivalence implies $\mathfrak{M}, w \forall \neq \beta(u)$, or $\mathfrak{M}_{w}, w \not \models \beta(u)$. Now, by Theorem 3.3, there is a color-preserving monotonic map $f$ from the unraveling $\left(\mathfrak{N}_{u}\right)_{\mathrm{t}}$ into $\mathfrak{M}_{w}$. Clearly $f(u)$ is a successor of $w$. Thus, by assumption and persistency, we have $\mathfrak{M}_{w}, f(u) \models \varphi$, which implies $\left(\mathfrak{N}_{u}\right)_{\mathrm{t}}, u \models \varphi$ as $\varphi \in \mathrm{MR}$. Hence $\mathfrak{N}, u \models \varphi$, as required.

Next, we generalize Theorem 3.3 by relaxing it from a statement about a color-preserving monotonic map into a model on a frame $\mathfrak{F}$ to a statement about a color-consistent monotonic map into the frame $\mathfrak{F}$. We call a monotonic map $f$ from an $n$-model $\mathfrak{N}$ into a frame $\mathfrak{F}=(W, R)$ color-consistent if for all points $w, u$ in $\mathfrak{N}$,

$$
f(w) R f(u) \Longrightarrow \operatorname{col}(w) \leq \operatorname{col}(u) .
$$

Theorem 3.7. Let $\mathfrak{F}$ be a (Kripke or descriptive) frame, and $\mathfrak{N}$ a finite rooted $n$-model. Then, $\mathfrak{F} \not \models \beta(\mathfrak{N})$ iff there is a color-consistent monotonic map from $\mathfrak{N}_{\mathrm{t}}$ into $\mathfrak{F}$.

Proof. The left to right direction follows from Theorem 3.3, as a colorpreserving monotonic map into a model is clearly color-consistent. For the other direction assume that $f$ is a monotonic color-consistent map from $\mathfrak{N}_{\mathrm{t}}$ into $\mathfrak{F}=(W, R, \mathcal{P})$.

Claim: For every $w$ in $\mathfrak{N}_{\mathrm{t}}$, there exists $U_{w} \in \mathcal{P}$ such that $f(w) \in U_{w}$, and for every other node $u$ in $\mathfrak{N}_{\mathrm{t}}, f(u) \in U_{w}$ iff $f(w) R f(u)$.

Proof of Claim. Define $U_{w}=\bigcap\left\{V_{u} \mid \neg f(w) R f(u)\right\}$, where $V_{u}$ is an upset in $\mathcal{P}$ containing $f(w)$ but not $f(u)$, whose existence is guaranteed by $\mathcal{P}$ being refined (which is the case also when $\mathfrak{F}$ is a Kripke frame).

Now, define $V(p)=\bigcup\left\{U_{w} \mid \mathfrak{N}_{\mathrm{t}}, w \models p\right\}$, where each $U_{w}$ is as in the claim. We verify that $f$ preserves colors between $\mathfrak{N}_{\mathrm{t}}$ and the model $(\mathfrak{F}, V)$, and $\mathfrak{F} \not \models \beta(\mathfrak{N})$ will then follow by Theorem 3.3. Now, by the definition of $V$ and the claim, $\mathfrak{N}_{\mathrm{t}}, w \models p$ implies $f(w) \in U_{w} \subseteq V(p)$, and thus $f(w) \in V(p)$. Conversely, if $f(u) \in V(p)$, then there exists $w$ such that $f(u) \in U_{w}$ and $\mathfrak{N}_{\mathrm{t}}, w \models p$. The former implies by the claim that $f(w) R f(u)$. Since $f$ is 
color-consistent, we have $\operatorname{col}(w) \leq \operatorname{col}(u)$, which implies that $\mathfrak{N}_{\mathrm{t}}, u \models p$, as required.

We end this section by deriving as an immediate consequence of the above theorem the surprising and important consequence that $\beta(\mathfrak{N})$-formulas are preserved by arbitrary substructures. We say that a (Kripke or descriptive) frame $\mathfrak{G}=\left(W^{\prime}, R^{\prime}, \mathcal{P}^{\prime}\right)$ is a substructure of another frame $\mathfrak{F}=(W, R, \mathcal{P})$ iff $W^{\prime} \subseteq W$ and $R^{\prime}=R \uparrow W^{\prime}$.

Corollary 3.8. Let $\mathfrak{F}$ and $\mathfrak{G}$ be (Kripke or descriptive) frames with $\mathfrak{G}$ a substructure of $\mathfrak{F}$. If $\mathfrak{F} \models \beta(\mathfrak{N})$ for some finite $n$-model $\mathfrak{N}$, then $\mathfrak{G} \models \beta(\mathfrak{N})$. In other words, validity of formulas in $B$ is preserved by substructures.

Proof. Suppose for contraposition that $\mathfrak{G} \not \models \beta(\mathfrak{N})$. By Theorem 3.7, there is a color-consistent monotonic map $f: \mathfrak{N}_{\mathrm{t}} \rightarrow \mathfrak{G}$. The map $f$ composed with the embedding from $\mathfrak{G}$ into $\mathfrak{F}$ is easily seen to be color-consistent. Thus, we conclude $\mathfrak{F} \not \models \beta(\mathfrak{N})$ by applying Theorem 3.7 again.

Not even in the descriptive case substructures impose any relation between the admissible sets of $\mathfrak{G}$ and $\mathfrak{F}$. This contrasts with the definition of the topo-subframes that are needed in modal logic. In modal logic the corresponding result does not apply, as a subframe logic like GL is an obvious counterexample. This property, together with Theorem 3.4, immediately implies that intermediate subframe logics are canonical. We will formally state this result and also generalize it to logics axiomatized by arbitrary NNIL- or MR-formulas in the next section in Corollaries 4.12 and 4.13 ,

\section{Universal models}

In this section we construct $n$-universal models for NNIL-formulas. This is a continuation of the project started in [29] in which among other things the 2universal model for NNIL was constructed using the subsimulations of [28]. In this present paper we will construct the universal models using colorpreserving monotonic maps instead. By the results of the previous section these models will actually also be universal models for MR-formulas. We will then be able to derive that NNIL-formulas are exactly the ones that are reflected by color-preserving monotonic maps, an important result occurring indirectly already in the earlier study of NNIL-formulas [28. We will also formally conclude, as a consequence of Corollary 3.8 in the previous section, that logics axiomatized by NNIL- or MR-formulas are canonical. 


\subsection{Definition and properties of universal models}

Universal models for modal logics and IPC were thoroughly investigated by a number of authors [1, 20, 15, 24, 25] (see [8, §8] and [3, §3] for an overview), and results for fragments of IPC can be found in [5, 17, 16, 22. We now give a formal and general definition for the notion of $n$-universal model that applies to rather different fragments of IPC in a uniform manner. Such a general definition will also allow us to point out the place and usefulness of universal models.

Definition 4.1. We say that a model $\mathfrak{M}=(W, R, V)$ is an $n$-universal model of a class $\Phi$ of $n$-formulas iff every upset generated by a single point in $W$ is finite, and the following conditions are satisfied:

(i) For any $\varphi, \psi \in \Phi$, if $\varphi \nvdash \psi$, then there exists $w \in W$ such that $w \models \varphi$ and $w \not \models \psi$.

(ii) For every upset $U$ of $\mathfrak{M}$ generated by a single point, there is $\varphi \in \Phi$ such that $V(\varphi)=U$.

Condition (i) in the $n$-universal model makes sure that "there are enough counterexamples", while condition (ii) ensures that "there are no superfluous" points. By taking the contrapositive of (i) we know that for any two formulas $\varphi, \psi \in \Phi$, if $V(\varphi) \subseteq V(\psi)$ in the universal model, then $\vdash \varphi \rightarrow \psi$; in particular, $V(\varphi)=V(\psi)$ implies $\vdash \varphi \leftrightarrow \psi$. Condition (ii) implies that no two distinct worlds in the $n$-universal model of $\Phi$ are $\Phi$-equivalent. For formula classes $\Phi$ that have the finite model property (e.g., for IPC), a model satisfying conditions (i) and (ii) automatically satisfies the requirement that point-generated upsets are finite. In general only for a locally finite formula class $\Phi$ do universal models seem to be useful.

The definable upsets in $n$-universal models will reflect the algebraic structure of the logic or fragment. In the case of IPC itself it will be the Lindenbaum-Tarski algebra, a Heyting Algebra, but this is also the case for the fragment of the formulas with only $\rightarrow$ and $\wedge$, because even without $\vee$ it forms a distributive lattice, which in the finite case always is a Heyting algebra. For the fragment of IPC with only $\rightarrow$ this is no longer the case but it still gives suitable $n$-universal models [22]. For the set of all NNIL-formulas in an arbitrary number of variables the Lindenbaum-Tarski algebra is not a Heyting algebra since, although they do form a distributive lattice, they are not closed under implication. But the NNIL-formulas in $n$ variables, by their local finiteness, do automatically form a Heyting algebra. 
Since these algebras are free algebras there is a close relationship with the $n$-canonical model (also known as $n$-Henkin model). Usually the $n$-universal model is the "upper part" of the $n$-Henkin model (see [3, 20]), and, in the case of locally finite fragments, isomorphic to it (see [5, 7]). Unsurprisingly, it will turn out in Theorem 4.14 that indeed the $n$-universal model is also in the NNIL-case isomorphic to the $n$-canonical model.

The mappings appropriate to the logic or the fragment play an important role in universal models. In general $n$-universal models have the property that any finite $n$-model is connected by such a map to a unique generated submodel of the universal model. This then gives a uniqueness property to the universal model and will imply that it is in a certain sense the smallest. For IPC itself these mappings are the p-morphisms, for NNIL their role will be taken over by the color-preserving monotonic maps.

All this means that it is often advantageous to see the $n$-universal model not as one model but as the collection of all of its point-generated submodels, ordered by the generated submodel relation. In fact, the latter was the only point of view in the proto-universal models of [11]. These different views of the model are pertinent in the case of the $n$-universal model for NNIL-formulas about to be constructed. In particular, every node in our $n$-universal model will be associated with a local tree that is mostly very different from the submodel generated by the node: the ordering of the local models is no longer the generated submodel relation, and thereby the isomorphism between the local model and the submodel of the universal model generated by it has been lost (although it can be recovered to a certain extent, see Proposition 4.7). An arbitrary finite $n$-tree will have a unique map to such a local tree in the $n$-universal model only. In fact, as we will show in Theorem 4.9, it can be monotonically mapped back and forth by color-preserving maps into that unique local tree.

Let us first briefly recall the construction of the $n$-universal model $\mathcal{U}(n)$ of IPC (see e.g. [20]). The construction of the $n$-universal model for NNILformulas to be given in Definition 4.3, though more complex, basically follows the same strategy.

Definition 4.2. The $n$-universal model $\mathcal{U}(n)=(W, R, V)$ of IPC is defined inductively in layers as follows.

- The first layer consists of nodes of the $2^{n}$ distinct $n$-colors.

- Assume that the $\leq m$ th layers have been defined already. We define the $(m+1)$ th layer as follows: 


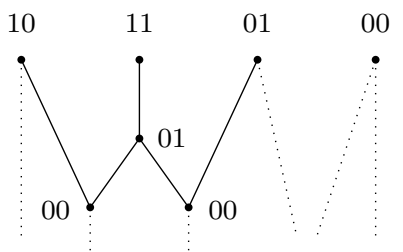

Figure 1: A fragment of $\mathcal{U}(2)$

- For each element $w$ in the $m$ th layer, and each color $c<\operatorname{col}(w)$, add a new node $u$ to layer $m+1$ with color $c$ and with $w$ the only immediate successor of $u$.

- For each set $X=\left\{w_{1}, \ldots, w_{k}\right\}(k \geq 2)$ of pairwise $R$-incomparable elements in layers $\leq m$ containing at least one member from layer $m$, and each color $c$ less than or equal to the color of all nodes in $X$, add a new node $w$ to layer $m+1$ with color $c$ and $w_{1}, \ldots, w_{k}$ as immediate successors.

The 1-universal model of IPC is also known as Rieger-Nishimura Ladder [21, 23]. See Figure 1 for a fragment of the 2-universal model $\mathcal{U}(2)$.

We now construct the $n$-universal model $\mathcal{T}(n)$ of NNIL-formulas. It will turn out that this model is also the $n$-universal model of MR-formulas. The nodes in our universal model $\mathcal{T}(n)$ will be finite tree-like models themselves, and we thus denote them as $T_{w}, T_{u}$, etc. The reader may think of $w, u$ as elements from $\mathcal{U}(n)$, and $T_{w}$ and $T_{u}$ as the unravelings of $\mathcal{U}(n)_{w}$ and $\mathcal{U}(n)_{u}$ to trees; in particular, the color of the root of $T_{w}$ is taken to be $\operatorname{col}(w)$. We will not use here other facts though concerning this "embedding" of $\mathcal{T}(n)$ into $\mathcal{U}(n)$. Each chain in the $T_{w}$ 's will be strictly decreasing in color, but not all such nodes in $\mathcal{U}(n)$ will participate in $\mathcal{T}(n)$. The ordering in our $\mathcal{T}(n)$ will not be the usual generated submodel ordering but the $\leq$ relation between models induced by color-preserving monotonic maps. We write $\mathfrak{M} \equiv \mathfrak{N}$ if both $\mathfrak{M} \leq \mathfrak{N}$ and $\mathfrak{N} \leq \mathfrak{M}$. It is easy to verify that the relation $\leq$ is reflexive and transitive, and thus $\equiv$ is an equivalence relation.

Definition 4.3. The $n$-model $\mathcal{T}(n)=(W, \leq, V)$ is defined as follows:

- The domain $W$ is defined inductively in layers:

- The first layer consists of nodes (or trees of single nodes) of the $2^{n}$ distinct $n$-colors. 
- Assume that the $\leq m$ th layers have been defined already. We define the $(m+1)$ th layer as follows: For every set $X=\left\{T_{w_{1}}, \ldots, T_{w_{k}}\right\}$ of pairwise $\leq$-incomparable trees in layers $\leq m$ containing at least one member of layer $m$, and every color $c$ strictly smaller than all the colors at the roots of the trees in $X$, build a tree $T_{w}$ by taking the disjoint union of the trees in $X$ and adding a fresh root $w$ of color $c$ below. Then add $T_{w}$ as a new node to layer $m+1$.

- Order (the trees in) $W$ by the $\leq$ relation.

- The color of a node $T_{w}$ in $W$ is defined as the color of the root $w$ in the tree $T_{w}$.

The colors in $\mathcal{T}(n)$ are easily seen to be persistent. In the next proposition we verify that $T(n)$ does not contain two $\equiv$-equivalent distinct nodes, which will imply that $\leq$ in $\mathcal{T}(n)$ is a partial order as it should be. See Figure 2 for $\mathcal{T}(2)$ with three layers: The first layer consists of all the singlepoint trees (indicated in the figure with the lightest shades). The second layer consists of all the nodes with darker shades, while the third layer consists of all the nodes with the darkest shades. An easy inductive argument shows that the $m$ th layer of $\mathcal{T}(n)$ contains trees $T_{w}$ only of depth $m$. On the other hand, since the relation of $\mathcal{T}(n)$ is the $\leq$ relation (instead of the usual generated submodel relation), the depth of a tree $T_{w}$ regarded as a node in $\mathcal{T}(n)$ is often larger, as demonstrated clearly in Figure 2 ,

Proposition 4.4. Let $T_{w}, T_{u} \in \mathcal{T}(n)$.

(1) If $f: T_{w} \rightarrow T_{w}$ is monotonic and color-preserving, then $f$ is the identity map on $T_{w}$.

(2) If $T_{w} \neq T_{u}$, then $T_{w} \not \equiv T_{u}$.

Proof. (1) We show the claim by induction on $d\left(T_{w}\right)$. If $d\left(T_{w}\right)=1$ the claim is obvious. Now let $d\left(T_{w}\right)>1$ and let $f: T_{w} \rightarrow T_{w}$ be a color-preserving monotonic map. Suppose $T_{w}$ is constructed from the set $X=\left\{T_{w_{1}}, \ldots T_{w_{k}}\right\}$ of pairwise $\leq$-incomparable trees in $\mathcal{T}(n)$ by adding a fresh root $w$ below. By construction no element in $X$ has a node of the color of $w$, thus we must have that $f(w)=w$, and $f\left(w_{i}\right) \neq w$ for each $1 \leq i \leq k$. The latter implies that for some $1 \leq j \leq k, f\left[T_{w_{i}}\right]$ is a submodel of $T_{w_{j}}$, or $T_{w_{i}} \leq T_{w_{j}}$, which can only happen when $T_{w_{i}}=T_{w_{j}}$, as distinct elements in $X$ are pairwise $\leq$-incomparable. This means that the restricted map $f \uparrow_{T_{w_{i}}}: T_{w_{i}} \rightarrow T_{w_{i}}$ is monotonic and color-preserving, and therefore the identity map by induction 


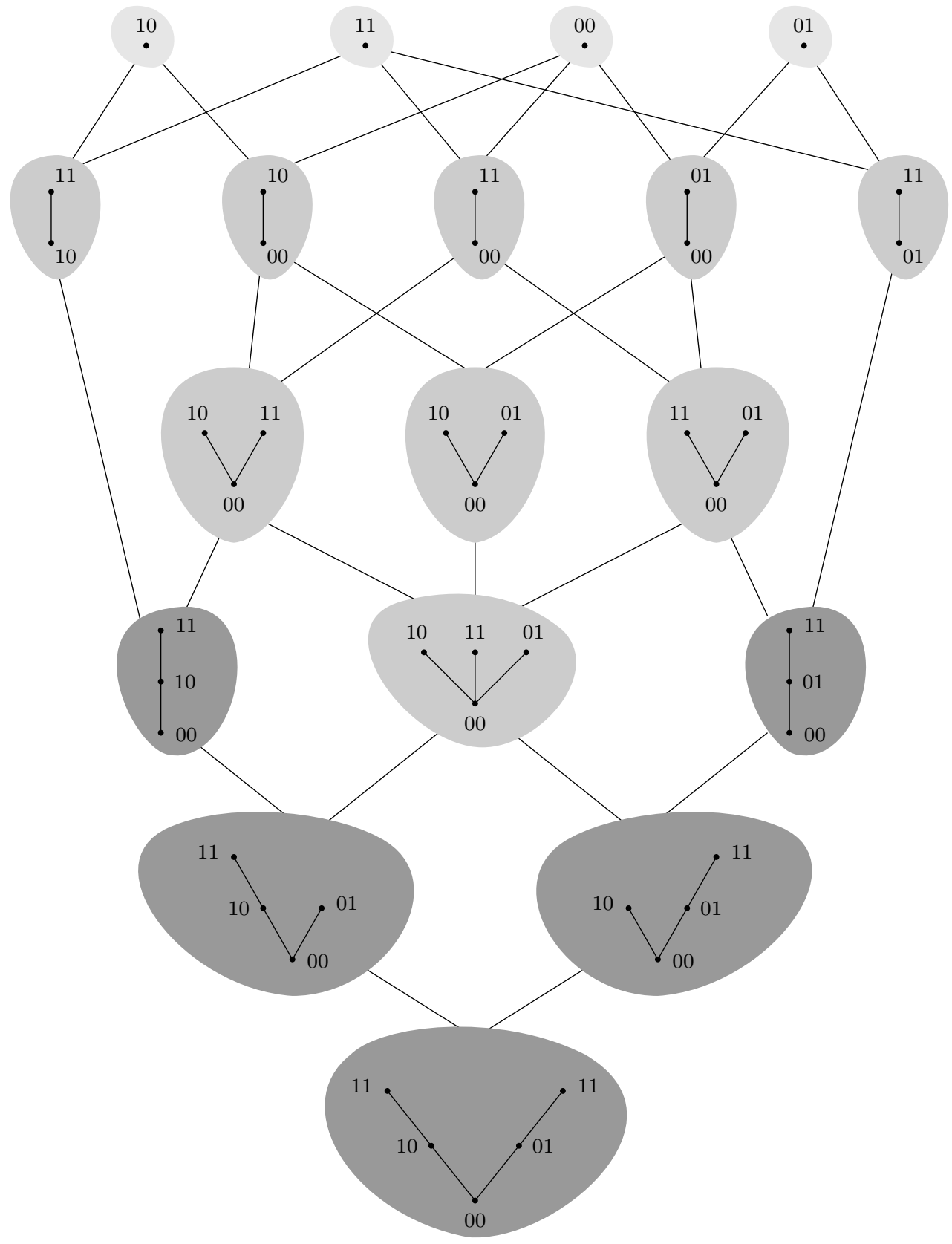

Figure 2: $\mathcal{T}(2)$ 
hypothesis. We have thus shown that $f$ restricted to all elements in $X$ is the identity map. Together with the fact that $f(w)=w$, we conclude that $f: T_{w} \rightarrow T_{w}$ itself is the identity map.

(2) Suppose $T_{w} \equiv T_{u}$, i.e. there are color-preserving monotonic maps $f: T_{w} \rightarrow T_{u}$ and $g: T_{u} \rightarrow T_{w}$. Then $g \circ f: T_{w} \rightarrow T_{w}$ and $f \circ g: T_{u} \rightarrow T_{u}$ are color-preserving monotonic maps, and thus the identity maps by item (11). So $g$ is a bijective monotonic map with a monotonic inverse. It is a well-known property of partial orders that in this case $T_{w}$ is isomorphic to $T_{u}$, and so $T_{w}=T_{u}$.

As we commented already, every tree $T_{w}$ in $\mathcal{T}(n)$ can be viewed as an unraveling of some generated submodel in $\mathcal{U}(n)$ with each chain strictly decreasing in color; for instance, the right immediate successor of the root of $\mathcal{T}(2)$ in Figure 2 corresponds to the submodel of $\mathcal{U}(2)$ generated by the node $w$ in Figure 1. Meanwhile, the submodel of $\mathcal{U}(2)$ generated by the node $u$, though with every chain strictly decreasing in color, is not present in $\mathcal{T}(2)$, because, e.g., the two subtrees generated by the two immediate successors of $u$ drawn in Figure 1 are not $\leq$-incomparable.

One crucial property of our $n$-universal model $\mathcal{T}(n)$ is that it is finite and rooted. This is clearly illustrated in Figure 2 of $\mathcal{T}(2)$, where the root is the universal 2-tree. Consider the $n$-model $\mathfrak{C}_{n}=\left(2^{n}, \leq, V_{n}\right)$ whose domain is the set of all $n$-colors ordered by the partial order $\leq$ of colors, and the color of a node is identical to the node itself. We call the universal $n$-tree, denoted by $T_{\mathbf{0}}$, the unraveling of $\mathfrak{C}_{n}$ with root $\mathbf{0}=0 \cdots 0$.

Fact 4.5. $\mathcal{T}(n)$ is finite and rooted with root $T_{\mathbf{0}}$.

Proof. We first show that $\mathcal{T}(n)$ is finite. Since every chain in a tree $T_{w}$ in $\mathcal{T}(n)$ is strictly decreasing in color, every tree $T_{w}$ has depth at most $n+1$, which also means that $\mathcal{T}(n)$ has at most $n+1$ layers. Each layer is also clearly finite, as essentially there are only finitely many $n$-colors strictly smaller than one fixed $n$-color.

Next, we show that $T_{\mathbf{0}}$ is in $\mathcal{T}(n)$. Clearly, every chain in $T_{\mathbf{0}}$ is strictly decreasing in color, and any two subtrees generated by nodes with the same parent are $\leq$-incomparable (as the two roots have different colors). Thus, an easy inductive argument shows that every generated subtree in every layer in $T_{\mathbf{0}}$ belongs to $\mathcal{T}(n)$, thereby $T_{\mathbf{0}}$ itself is in $\mathcal{T}(n)$.

Finally, we show that $T_{\mathbf{0}}$ is the root of $\mathcal{T}(n)$, that is, $T_{\mathbf{0}} \leq T_{w}$ for every tree $T_{w}$ in $\mathcal{T}(n)$. First note that every two subtrees of $T_{\mathbf{0}}$ generated by two points of the same color $c$ are isomorphic, as they are both the unraveling of the submodel of $\mathfrak{C}_{n}$ generated by $c$. One can then easily show by induction 
on the depth of $T_{w}$ that $T_{c} \leq T_{w}$ for any subtree $T_{c}$ of $T_{\mathbf{0}}$ generated by a point $c$ with the same color as $w$. Since $T_{\mathbf{0}} \leq T_{c}$, we obtain finally $T_{\mathbf{0}} \leq T_{w}$.

As pointed out already, the trees $T_{w}$ are in general not isomorphic to the submodel of $\mathcal{T}(n)$ generated by the node $T_{w}$. But every node $v$ in a tree $T_{w}$ does generate a tree $T_{v}$ from $\mathcal{T}(n)$, as the following fact shows.

Fact 4.6. If $T_{w} \in \mathcal{T}(n)$ and $v \in T_{w}$, then the subtree $T_{v}$ of $T_{w}$ belongs to $\mathcal{T}(n)$.

Proof. We prove the fact by induction on the layer in $\mathcal{T}(n)$ that $T_{w}$ belongs to. If $T_{w}$ is a tree of a single node in the first layer, the fact trivially holds. If $T_{w}$ is in layer $m+1$, then by the construction of $\mathcal{T}(n)$, every immediate successor $v$ of $w$ in $T_{w}$ generates a tree $T_{v}$ in $\mathcal{T}(n)$. Since $T_{v}$ is in layer $\leq m$, by induction hypothesis, every node $u$ in $T_{v}$ generates a tree $T_{u}$ in $\mathcal{T}(n)$.

Since the nodes in $\mathcal{T}(n)$ are models themselves, a formula $\varphi$ can be evaluated at a point $T_{w}$ of $\mathcal{T}(n)$ in two ways: Either in the model $T_{w}$ (at its root $w$ ), or in the universal model $\mathcal{T}(n)$ at the node $T_{w}$. The next proposition shows that the truth values of NNIL- or MR-formulas $\varphi$ in $n$ variables for both ways of evaluation actually coincide. Hereafter we will then use the notation $T_{w} \models \varphi$ for either $\mathcal{T}(n), T_{w} \models \varphi$ or $T_{w}, w \models \varphi$.

Proposition 4.7. For any $T_{w} \in \mathcal{T}(n)$, we have $\left(T_{w}, w\right) \simeq_{\mathrm{NNIL}}\left(\mathcal{T}(n), T_{w}\right)$, and thus $\left(T_{w}, w\right) \simeq_{\mathrm{MR}}\left(\mathcal{T}(n), T_{w}\right)$ as well.

Proof. Since $\mathcal{T}(n)$ and $T_{w}$ are finite, by Proposition 3.6, the result for MRformulas follows from that for NNIL-formulas. We now prove by induction that $T_{w}, w \models \varphi$ iff $\mathcal{T}(n), T_{w} \models \varphi$ for any NNIL-formula $\varphi$ in $n$ variables in normal form.

If $\varphi=\perp$, the claim holds trivially. If $\varphi=p$, the claim also holds since $\operatorname{col}(w)=\operatorname{col}\left(T_{w}\right)$. The induction steps for $\wedge$ and $\vee$ are easy. We now prove the claim for the case $\varphi=p \rightarrow \psi$.

Suppose $T_{w}, w \models p \rightarrow \psi$. To show that $\mathcal{T}(n), T_{w} \models p \rightarrow \psi$ let $T_{u} \in \mathcal{T}(n)$ be such that $T_{w} \leq T_{u}$ and $\mathcal{T}(n), T_{u} \models p$. Then we obtain $T_{u}, u \models p \rightarrow \psi$ by Lemma 2.2. Moreover, since $\operatorname{col}(u)=\operatorname{col}\left(T_{u}\right)$, we have $T_{u}, u \models p$, which then implies $T_{u}, u \models \psi$. Thus, we conclude by induction hypothesis that $\mathcal{T}(n), T_{u} \models \psi$, as required.

Conversely, suppose $\mathcal{T}(n), T_{w} \models p \rightarrow \psi$. Let $u \in T_{w}$ be such that $T_{w}, u \models$ $p$. By Fact 4.6, we know that $T_{u} \in \mathcal{T}(n)$. Since $\operatorname{col}(u)=\operatorname{col}\left(T_{u}\right)$, we have 
$\mathcal{T}(n), T_{u}=p$. As the identity map from $T_{u}$ into $T_{w}$ is monotonic and colorpreserving, we also have $T_{w} \leq T_{u}$. It then follows that $\mathcal{T}(n), T_{u} \models \psi$, which, by induction hypothesis, implies that $T_{u}, u \models \psi$. Again since $T_{w} \leq T_{u}$, we obtain $T_{w}, u \models \psi$ by Lemma 2.2, and hence $T_{w}, w \models p \rightarrow \psi$.

One can view the trees $T_{w}$ in $\mathcal{T}(n)$ as representatives of $\equiv$-equivalence classes of $n$-trees, in the sense that every finite tree-like $n$-model is $\equiv$ equivalent to some (unique) tree $T_{w}$ in $\mathcal{T}(n)$. We now prove this important property of $\mathcal{T}(n)$.

Lemma 4.8. For every finite $n$-tree $\mathfrak{T}$, there is a node $T_{w}$ in $\mathcal{T}(n)$ such that

(1) $T_{w} \leq_{f} \mathfrak{T}$ via some surjective color-preserving monotonic map $f$,

(2) $T_{w}$ is isomorphic to a submodel of $\mathfrak{T}$ that has the same root as $\mathfrak{T}$,

Proof. We prove the lemma by the number of different colors in $\mathfrak{T}$. If all nodes in $\mathfrak{T}$ have one and the same color $c$, then the tree $T_{w}$ in $\mathcal{T}(n)$ consisting of a single node with the color $c$ clearly satisfies conditions (1) and (2).

Now assume that the nodes in $\mathfrak{T}$ have at least two different colors. Let $x_{1}, \ldots, x_{m}$ be the minimal nodes in $\mathfrak{T}$ with a color different from the root $r$, and let $\mathfrak{T}_{1}, \ldots, \mathfrak{T}_{m}$ be the subtrees in $\mathfrak{T}$ generated by these nodes respectively. Applying the induction hypothesis to these subtrees we get the corresponding trees $T_{w_{1}}, \ldots, T_{w_{m}}$ in $\mathcal{T}(n)$ satisfying conditions (1) and (2). Assume without loss of generality that $T_{w_{1}}, \ldots, T_{w_{k}}$ are the minimal elements among $T_{w_{1}}, \ldots, T_{w_{m}}$ with respect to $\leq$ (and are therefore pairwise incomparable). Let $T_{w}$ be the tree formed by taking the disjoint union of $T_{w_{1}}, \ldots, T_{w_{k}}$ and adding a root $w$ below with $\operatorname{col}(r)$ (which is strictly less than all colors occurring in each $\left.T_{w_{i}}\right)$. Clearly $T_{w}$ is a node in $\mathcal{T}(n)$. We now verify that $T_{w}$ satisfies conditions (1) and (2).

To see condition (2), by induction hypothesis each $T_{w_{i}}$ is isomorphic to a submodel of $\mathfrak{T}_{i}$ with root $x_{i}$, and $\operatorname{col}(w)=\operatorname{col}(r)$. Thus the tree $T_{w}$ is isomorphic to a submodel of $\mathfrak{T}$ with root $r$.

For condition (1), first for each $1 \leq i \leq m$, there is a color-preserving monotonic map $f_{i}$ from $\mathfrak{T}_{i}$ onto $T_{w_{i}}$ given by induction hypothesis. Also, for each $k+1 \leq i \leq m$, there is $1 \leq j_{i} \leq k$ such that $T_{w_{j_{i}}} \leq T_{w_{i}}$ via a color-preserving monotonic map $g_{i}: T_{w_{i}} \rightarrow T_{w_{j_{i}}}$. Now, we define a map $f: \mathfrak{T} \rightarrow T_{w}$ by taking

$$
f(x)= \begin{cases}w & \text { if } \operatorname{col}(x)=\operatorname{col}(r) \\ f_{i}(x) & \text { if } x \in \mathfrak{T}_{i} \text { for some } i \leq k ; \\ g_{i} \circ f_{i}(x) & \text { if } x \in \mathfrak{T}_{i} \text { for some } k+1 \leq i \leq m .\end{cases}
$$


It is easy to see that $f$ is monotonic, color-preserving and onto.

Theorem 4.9. For every finite $n$-tree $\mathfrak{T}$, there is a unique node $T_{w}$ in $\mathcal{T}(n)$ with $\mathfrak{T} \equiv T_{w}$, and thus $\mathfrak{T} \simeq_{\mathrm{MR}} T_{w}$.

Proof. Let $T_{w}$ be the tree in $\mathcal{T}(n)$ given by Lemma 4.8. Then the two conditions in Lemma 4.8 imply immediately $\mathfrak{T} \equiv T_{w}$. The uniqueness of $T_{w}$ follows from Proposition 4.4(2).

Next, we verify that $\mathcal{T}(n)$ satisfies the two conditions in Definition 4.1 of universal models with respect to NNIL- or MR-formulas. Since NNILformulas are also MR-formulas, we can then conclude that $\mathcal{T}(n)$ is a universal model for both NNIL- and MR-formulas. Moreover, we show that $\mathcal{T}(n)$ is actually an exact model for NNIL- and MR-formulas, which is a universal model with the second condition strengthened to "for all upsets $U$ of $\mathfrak{M}$ (not necessarily generated by single points), there is $\varphi \in \Phi$ such that $V(\varphi)=$ $U$ " (see [22]). Note that infinite universal models (such as the $n$-universal models for IPC) are in general not exact, whereas $n$-universal models for locally finite fragments of IPC often are $([16,22])$.

Proposition 4.10. (1) For any $n$-formulas $\varphi, \psi \in \mathrm{MR}$, if $\varphi \nvdash \psi$, then there exists a node in $\mathcal{T}(n)$ verifying $\varphi$ and falsifying $\psi$.

(2) For each upset $U$ of $\mathcal{T}(n)$, there exists a NNIL-formula $\beta^{+}(U)$ such that for each $T_{u} \in \mathcal{T}(n), T_{u} \models \beta^{+}(U)$ iff $T_{u} \in U$.

In particular, for each node $T_{w}$ in $\mathcal{T}(n)$, there exists a NNIL-formula $\beta^{+}(w)$ such that for each $T_{u} \in \mathcal{T}(n), T_{u} \models \beta^{+}(w)$ iff $T_{w} \leq T_{u}$.

Proof. (1) If $\varphi \not \forall \psi$, then there is a finite tree $\mathfrak{T}$ verifying $\varphi$ and refuting $\psi$. By Theorem 4.9, there is a node $T_{w}$ in $\mathcal{T}(n)$ with the same property.

(2) Define $\beta^{+}(U)=\bigwedge\left\{\beta(v) \mid T_{v} \in \mathcal{T}(n)\right.$ and $\left.T_{v} \notin U\right\}$. Suppose $T_{u} \in U$. For any $T_{v} \in \mathcal{T}(n)$ with $T_{v} \notin U$, we have $T_{u} \not \leq T_{v}$, which implies, by Theorem [3.3, that $T_{u} \models \beta(v)$. Hence $T_{u} \models \beta^{+}(U)$.

Conversely, suppose $T_{u} \notin U$. Then $\beta(u)$ is a conjunct in $\beta^{+}(U)$, and since $T_{u} \not \models \beta(u)$ by Lemma 3.2, we obtain $T_{u} \not \models \beta^{+}(w)$.

Now that we have proved all these concrete properties of $\mathcal{T}(n)$ let us remark that there are alternative ways to see the universal model $\mathcal{T}(n)$. For instance, since $\mathcal{T}(n)$ and $\mathcal{U}(n)$ are constructed basically by the same strategy (see Definitions 4.2 and 4.3), one can view $\mathcal{T}(n)$ as the set of those unraveled elements $T$ of $\mathcal{U}(n)$ with only strictly color-decreasing chains such that for every node $w$ in $T$, the trees $T_{w_{i}}$ generated by immediate successors $w_{i}$ of 
$w$ are pairwise $\leq$-incomparable. Indeed, it is easy to see by induction that each such unraveled element $T$ of $\mathcal{U}(n)$ belongs to $\mathcal{T}(n)$, and conversely each element in $\mathcal{T}(n)$ is an unraveled element $T$ of $\mathcal{U}(n)$ of the described form.

\subsection{Consequences of the universal model}

Having seen that $\mathcal{T}(n)$ is the universal model for both NNIL- and MRformulas, we now derive in the following three corollaries an even closer relationship of NNIL-formulas with MR-formulas as well as the class B of NNIL-subframe formulas we defined earlier. First, we conclude that NNILformulas are exactly the ones reflected by color-preserving monotonic maps, a result essentially already following from [28].

Corollary 4.11. For any MR-formula $\varphi$, there is a finite set $\mathrm{B}_{\varphi} \subseteq \mathrm{B}$ of NNILsubframe formulas such that $\vdash \varphi \leftrightarrow \bigwedge_{\beta(w) \in \mathrm{B}_{\varphi}} \beta(w)$. In particular, every MR-formula is equivalent to a NNIL-formula, and NNIL-formulas are (up to equivalence) exactly the formulas reflected by color-preserving monotonic maps.

Proof. Consider the upset $V(\varphi)$ in the universal model $\mathcal{T}(n)$. By (the proof of) Proposition 4.10(2) there is a finite set $\mathrm{B}_{\varphi} \subseteq \mathrm{B}$ of NNIL-subframe formulas such that $T_{u} \models \bigwedge_{\beta(w) \in \mathrm{B}_{\varphi}} \beta(w)$ iff $T_{u} \models \varphi$ for every $T_{u} \in \mathcal{T}(n)$. Thus $\vdash_{\mathrm{IPC}} \varphi \leftrightarrow \bigwedge_{\beta(w) \in \mathrm{B}_{\varphi}} \beta(w)$ by the property of universal model.

Next we infer that the intermediate logics axiomatized by B-, MR-, or NNIL-formulas coincide. Therefore, by Theorem 3.4, formulas from one and any of the three classes are sufficient to axiomatize all subframe logics.

Corollary 4.12. For an intermediate logic $L$, the following are equivalent:

(1) $L$ is axiomatizable by B-formulas;

(2) $L$ is axiomatizable by NNIL-formulas;

(3) $L$ is axiomatizable by MR-formulas;

(4) $L$ is a subframe logic.

Proof. By Theorem 3.4, (4) is equivalent to (2). Since B $\subseteq$ NNIL $\subseteq$ MR, the implications $(3) \Rightarrow(2) \Rightarrow(1)$ are obvious. By Corollary 4.11 every MRformula is equivalent to a set of B-formulas, thus (1) implies (3) follows. 
This corollary allows us to complete the discussion from the ending of the previous section and to conclude surprisingly easily to the well-known fact that subframe logics $L$ are canonical, i.e., the underlying Kripke frames of the canonical models of $L$ are $L$-frames.

Corollary 4.13. The class of frames of any subframe logic is closed under taking substructures. In particular, subframe logics are canonical.

Proof. The first part follows immediately from Corollary 3.8 and the equivalence of items (1) and (4) in Corollary 4.12.

For any subframe logic $L$, consider its canonical model. The descriptive frame of its definable upsets validates $L$. Since the underlying Kripke frame of this descriptive $L$-frame is obviously a substructure, it validates $L$, i.e., $L$ is canonical.

Finally, let us conclude our discussion on $n$-universal models $\mathcal{T}(n)$ for NNIL-formulas by showing that they are isomorphic to $n$-canonical models for NNIL-formulas. Let $\mathrm{NNIL}_{n}$ denote the class of all NNIL-formulas in $n$ variables, and note that up to equivalence this class is finite since NNILformulas are locally finite. Recall that the $n$-canonical mode $\mathfrak{M}_{\mathrm{NNIL}_{n}}$ for $\mathrm{NNIL}_{n}$-formulas is constructed in the standard manner (as in the case of full IPC): Elements in $\mathfrak{M}_{\mathrm{NNIL}_{n}}$ are the consistent theories of $\mathrm{NNIL}_{n}$-formulas (i.e., sets $\Gamma$ of $\mathrm{NNIL}_{n}$-formulas such that $\Gamma \nvdash \perp$, and $\Gamma \vdash \varphi$ implies $\varphi \in \Gamma$ for all $\varphi \in \mathrm{NNIL}_{n}$ ) with the disjunction property (i.e., $\varphi \vee \psi \in \Gamma$ implies either $\varphi \in \Gamma$ or $\psi \in \Gamma$ ), and the ordering is the set inclusion relation $\subseteq$. Observe that since NNIL-formulas are locally finite, a theory $\Gamma$ of $\mathrm{NNIL}_{n^{-}}$ formulas is (modulo equivalence) a finite set $\Gamma_{0}$ or a single formula $\varphi=\bigwedge \Gamma_{0}$. Elements of $\mathfrak{M}_{\mathrm{NNIL}_{n}}$ are thus actually the theories generated by the (single and consistent) $\mathrm{NNIL}_{n}$-formulas $\varphi$ with the disjunction property (i.e., $\varphi \vdash$ $\psi \vee \chi$ implies $\varphi \vdash \psi$ or $\varphi \vdash \chi)$. The $\mathrm{NNIL}_{n}$-formulas axiomatizing the theories in $\mathfrak{M}_{\mathrm{NNIL}_{n}}$ are in fact the $\beta^{+}(w)$ formulas defined in Proposition 4.10)(2), as will follow from the next theorem.

Theorem 4.14. $\mathfrak{M}_{\mathrm{NNIL}_{n}}$ (with relation $\subseteq$ ) is isomorphic to $\mathcal{T}(n)$ (with relation $\leq)$.

Proof. Define a function $f$ from $\mathcal{T}(n)$ into $\mathfrak{M}_{\mathrm{NNIL}_{n}}$ by taking

$$
f\left(T_{w}\right)=\operatorname{Th}\left(T_{w}\right)=\left\{\varphi \in \mathrm{NNIL}_{n} \mid T_{w} \models \varphi\right\} .
$$

Each $\operatorname{Th}\left(T_{w}\right)$ clearly has the disjunction property, and thus $f$ is well-defined. Also, obviously $f$ preserves colors. By property (ii) of the universal model 
(Definition 4.1), the theories of two distinct points in $\mathcal{T}(n)$ differ, and thus $f$ is injective.

To see that $f$ is onto take any $\Phi \in \mathfrak{M}_{\mathrm{NNIL}_{n}}$, and let $\varphi$ axiomatize $\Phi$. Let $\psi_{1}, \ldots, \psi_{k}$ contain a member of each equivalence class in $\mathrm{NNIL}_{n} \backslash \Phi$. Clearly $\Phi \nvdash \psi_{i}$ for all $i$, as $\Phi$ is a theory. It then follows that $\Phi \nvdash \bigvee_{i} \psi_{i}$, as $\Phi$ has the disjunction property. Now, by property (i) of the universal model (or Proposition 4.10(1)), there is some node $T_{w}$ in $\mathcal{T}(n)$ verifying $\varphi$ and refuting $\bigvee_{i} \psi_{i}$. This means that $\Phi=\operatorname{Th}\left(T_{w}\right)=f\left(T_{w}\right)$, as desired.

It remains to show that $f$ is two ways order-preserving. Clearly $T_{w} \leq T_{u}$ implies $\operatorname{Th}\left(T_{w}\right) \subseteq \operatorname{Th}\left(T_{u}\right)$. Conversely, if $T_{w} \not \leq T_{u}$, by Theorem 3.3 we have $T_{w} \models \beta(u)$. Meanwhile, by Lemma 3.2 we have $T_{u} \not \models \beta(u)$. Hence $\operatorname{Th}\left(T_{w}\right) \nsubseteq \mathbb{T h}\left(T_{u}\right)$.

\section{$5 \quad$ Finite color-preserving submodels and the finite model property}

In this section, we give an alternative and direct proof that logics axiomatized by NNIL-formulas (i.e., all subframe logics) have the finite model property. Our proof is quite different from the previous proofs like the one of Theorem 11.20 in [8], which uses canonical formulas. We will introduce a procedure to reduce infinite models to finite submodels in a color-preserving manner. In case the infinite model is tree-like the finite reduced model is indistinguishable from it by MR- or NNIL-formulas. Our central argument then relies heavily on the result that logics axiomatized by NNIL-formulas are preserved under substructures (Corollaries 4.12 and 4.13 ). In the proof we also make use of a folklore frame-normal form of IPC-formulas, for which we include in the paper a direct semantic proof.

Let us start by defining the notion of color-preserving submodel that will play an important role in our construction.

Definition 5.1. A submodel $\mathfrak{N}=\left(W_{0}, R, V\right)$ of a model $\mathfrak{M}=(W, R, V)$ is said to be color-preserving, denoted $\mathfrak{N} \subseteq \subseteq_{c} \mathfrak{M}$, if, for any $w \in W_{0}$ and $u \in W$, $w R u$ implies that there exists $v \in W_{0}$ such that $w R v$ and $\operatorname{col}(v)=\operatorname{col}(u)$.

The above notion is related to the concept of relatively open introduced by Ghilardi in [14]. A function $f: \mathfrak{N} \rightarrow \mathfrak{M}$ is relatively open with respect to $g$ (also $g$-open) if, whenever $f(w) R u$, there exists $u^{\prime}$ in $\mathfrak{N}$ such that $w R u^{\prime}$ and $g\left(f\left(u^{\prime}\right)\right)=g(u)$. Observe that $\mathfrak{N} \subseteq \subseteq_{c} \mathfrak{M}$ iff the inclusion map $i: \mathfrak{N} \rightarrow \mathfrak{M}$ is col-open. It would be possible to reformulate proofs in this section, 
especially the proof of Theorem 5.8, using the properties of relatively open maps.

In the next lemma we prove some basic facts concerning color-preserving submodels.

Lemma 5.2. (1) If $\mathfrak{M}_{0} \subseteq_{c} \mathfrak{M}_{1}$ and $\mathfrak{M}_{1} \subseteq_{c} \mathfrak{M}_{2}$, then $\mathfrak{M}_{0} \subseteq_{c} \mathfrak{M}_{2}$.

(2) Let $\alpha$ be a p-morphism from $\mathfrak{M}$ into $\mathfrak{M}^{\prime}$. If $\mathfrak{N} \subseteq_{c} \mathfrak{M}$, then $\alpha[\mathfrak{N}] \subseteq_{c} \mathfrak{M}^{\prime}$, where $\alpha[\mathfrak{N}]$ is the image of $\mathfrak{N}$ under $\alpha$.

Proof. The proofs for both items are standard. We only give the proof of item (2). Let $\mathfrak{M}=(W, R, V), \mathfrak{M}^{\prime}=\left(W^{\prime}, R^{\prime}, V^{\prime}\right)$ and $\mathfrak{N}=\left(W_{0}, R, V\right)$. Let $w \in W_{0}$ and $u^{\prime} \in W^{\prime}$ be such that $\alpha(w) R^{\prime} u^{\prime}$. Since $\alpha$ is a p-morphism, there exists $u \in W$ such that $\alpha(u)=u^{\prime}$ and $w R u$. By assumption, $\mathfrak{N}$ is a color-preserving submodel of $\mathfrak{M}$, thus there exists $v \in W_{0}$ such that $w R v$ and $\operatorname{col}(v)=\operatorname{col}(u)$. It follows that for $\alpha(v)$ in $\alpha[\mathfrak{N}]$, we have $\alpha(w) R^{\prime} \alpha(v)$ and $\operatorname{col}(\alpha(v))=\operatorname{col}(v)=\operatorname{col}(u)=\operatorname{col}(\alpha(u))=\operatorname{col}\left(u^{\prime}\right)$, as required.

We now proceed to reduce an infinite model to a finite one in a colorpreserving manner in a number of steps. In the first two steps we do this with trees, first reducing the depth of a tree-like model to finite, and in the next step the width. In the third step, in Theorem [5.5, we adapt this to infinite models in general, connect it with color-preserving monotonic maps, and show that validity of MR- and NNIL-formulas is preserved by the operation.

Lemma 5.3. Every tree-like $n$-model $\mathfrak{M}$ has a tree-like color-preserving submodel $\mathfrak{N}$ of finite depth with the same root.

Proof. Let $\mathfrak{M}=(W, R, V)$ and let $r$ be the root. Since $\mathfrak{M}$ is a tree, every node $w$ above $r$ has a unique immediate predecessor that we denote by $w_{0}$. Let $\mathfrak{N}$ be the submodel of $\mathfrak{M}$ on the set

$$
W_{0}=\{r\} \cup\left\{w \in W \mid \operatorname{col}\left(w_{0}\right)<\operatorname{col}(w)\right\} .
$$

The model $\mathfrak{N}$ has finite depth since all chains in $\mathfrak{N}$ are strictly increasing in color and there are only finitely many $n$-colors.

It remains to check that $\mathfrak{N}$ is a color-preserving submodel of $\mathfrak{M}$. For any $w \in W_{0}$ and $u \in W$ such that $w R u$, since $\operatorname{col}\left(w_{0}\right)<\operatorname{col}(w) \leq \operatorname{col}(u)$, there must exist a node $v$ in the finite set $R(w) \cap R^{-1}(u)$ such that $\operatorname{col}\left(v_{0}\right)<$ $\operatorname{col}(v)=\operatorname{col}(u)$. Clearly, $v \in W_{0}$ and $w R v$.

Next, we prune a tree of finite depth further to one of finite width, and thus obtain a finite tree. 
Lemma 5.4. Every tree-like $n$-model $\mathfrak{M}$ of finite depth has a finite tree-like color-preserving submodel $\mathfrak{N}$ with the same root.

Proof. Assume that $\mathfrak{M}$ is of depth $k$. We inductively select a sequence of color-preserving submodels $\mathfrak{N}_{k} \subseteq_{c} \cdots \subseteq_{c} \mathfrak{N}_{2} \subseteq_{c} \mathfrak{N}_{1}=\mathfrak{M}$ from $\mathfrak{M}$ such that in each $\mathfrak{N}_{i}$, there are only finitely many non-isomorphic subtrees generated by every node of depth $i$. This way each layer of $\mathfrak{N}_{k}$ and thus $\mathfrak{N}_{k}$ itself will be finite.

To construct $\mathfrak{N}_{2}$ consider the nodes $w$ of depth 2 . Each such $w$ has only maximal nodes as its immediate successors. These immediate successors have at most $2^{n}$ color types, and of each color type we keep only one immediate successor of $w$ and remove all the others. Clearly, after the reduction there are only finitely many non-isomorphic subtrees generated by the nodes $w$ of depth 2 , as these $w$ can have at most $2^{n}$ distinct colors.

Now assume that $\mathfrak{N}_{i}$ has been defined. We construct $\mathfrak{N}_{i+1}$ by deleting some subtrees. Consider an arbitrary node $w$ of depth $i+1$. By induction, there are only finitely many non-isomorphic subtrees generated by the immediate successors of $w$. Of each such isomorphism type we keep only one subtree above $w$ and remove all the others. Clearly, after the reduction there are only finitely many non-isomorphic subtrees generated by the nodes $w$ of depth $i+1$, as, again, these $w$ can have at most $2^{n}$ distinct colors.

Finally, we verify that $\mathfrak{N}_{k}$ is a color-preserving submodel of $\mathfrak{M}$ by proving that $\mathfrak{N}_{i+1}=\left(W_{i+1}, R, V\right)$ is a color-preserving submodel of $\mathfrak{N}_{i}=\left(W_{i}, R, V\right)$ for each $i$. Suppose $w \in W_{i+1}, u \in W_{i}$ and $w R u$. If $u \in W_{i+1}$, then we are done. Otherwise, $u$ is in a subtree $T$ of $\mathfrak{N}_{i}$ that is missing in $\mathfrak{N}_{i+1}$. By the construction there remains an isomorphic copy of $T$ in $\mathfrak{N}_{i+1}$ above $w$ and the node corresponding to $u$ in this isomorphic copy will have the same color as $u$. So we are also done.

The construction in this lemma is quite close to constructing the bisimulation quotient of $\mathfrak{M}$ but here it is relativized to trees. A similar construction occurs already in [11].

Theorem 5.5. Every rooted $n$-model $\mathfrak{M}$ has a finite color-preserving submodel $\mathfrak{N}$ with the same root.

In addition, if $\mathfrak{M}$ is tree-like, then $\mathfrak{N} \leq_{f} \mathfrak{M}$ for some surjective map $f$, and so $(\mathfrak{M}, r) \simeq_{\mathrm{MR}}(\mathfrak{N}, r)$ and $(\mathfrak{M}, r) \simeq_{\mathrm{NNIL}}(\mathfrak{N}, r)$.

Proof. We construct $\mathfrak{N}$ in stages. First unravel $\mathfrak{M}$ to obtain a tree-like model $\mathfrak{M}_{\mathrm{t}}$ with the same root. Second, apply Lemma 5.3 to $\mathfrak{M}_{\mathrm{t}}$ to obtain a treelike color-preserving submodel of finite depth with the same root. Lemma 
5.4 then gives a finite tree-like color-preserving submodel $\mathfrak{N}_{0}$ with the same root. Then, by Lemma $5.2(1), \mathfrak{N}_{0}$ is a color-preserving submodel of $\mathfrak{M}_{\mathrm{t}}$. Let $\alpha$ be the natural p-morphism from $\mathfrak{M}_{\mathrm{t}}$ onto $\mathfrak{M}$. By Lemma $5.2(2)$, the image $\mathfrak{N}=\alpha\left[\mathfrak{N}_{0}\right]$ of the finite model $\mathfrak{N}_{0}$ under $\alpha$ is a finite color-preserving submodel of $\mathfrak{M}$. Since $\alpha$ maps the root of $\mathfrak{M}_{\mathrm{t}}$ to the root of $\mathfrak{M}, \mathfrak{N}$ and $\mathfrak{M}$ have the same root.

Now, suppose in addition that $\mathfrak{M}$ is tree-like. Then $\mathfrak{N}$ can be obtained directly from $\mathfrak{M}$ by subsequently applying Lemma 5.3 and Lemma 5.4. We show that $\mathfrak{N}$ is also a monotonic image of $\mathfrak{M}$.

Let $\mathfrak{N}_{1}$ be the model of depth $k$ obtained from $\mathfrak{M}$ as in Lemma 5.3, For each $w$ in $\mathfrak{M}$, by the construction (and using the same notation) there is a predecessor $w^{\prime}$ of $w$ in $\mathfrak{N}_{1}$ such that $\operatorname{col}\left(w_{0}^{\prime}\right)<\operatorname{col}\left(w^{\prime}\right)=\operatorname{col}(w)$. Clearly the map $g$ from $\mathfrak{M}$ into $\mathfrak{N}_{1}$ defined as $g(w)=w^{\prime}$ is monotonic, color-preserving and onto.

Let $\mathfrak{N}=\mathfrak{N}_{k} \subseteq_{c} \cdots \subseteq_{c} \mathfrak{N}_{2} \subseteq_{c} \mathfrak{N}_{1}$ be the sequence of models as constructed in Lemma 5.4. We define maps $g_{i}: \mathfrak{N}_{i} \rightarrow \mathfrak{N}_{i+1}$ for every $1 \leq i \leq k-1$ as follows. The map $g_{i}$ sends a subtree that is removed in the construction to its isomorphic copy that is kept in $\mathfrak{N}_{i+1}$. Each $g_{i}$ is clearly monotonic and onto (and in fact it is a p-morphism). Finally, the composition $g_{k-1} \circ \cdots \circ g_{1} \circ g$ is a color-preserving monotonic map from $\mathfrak{M}$ onto $\mathfrak{N}$.

Lastly, together with the fact that the identity map from the submodel $\mathfrak{N}$ into $\mathfrak{M}$ is monotonic, we conclude that $(\mathfrak{M}, r) \simeq_{\mathrm{MR}}(\mathfrak{N}, r)$ and $(\mathfrak{M}, r) \simeq_{\mathrm{NNIL}}$ $(\mathfrak{N}, r)$ by definition of the class MR and Lemma 2.2 for NNIL-formulas.

As a simple application of the above theorem, we can show that Theorem 4.9 holds in case $\mathfrak{T}$ is an infinite tree as well, because $\mathfrak{T}$ can be reduced to a finite tree-like submodel $\mathfrak{T}_{0}$ that is a monotonic image of $\mathfrak{T}$, in particular, $\mathfrak{T}_{0} \equiv \mathfrak{T}$.

In the rest of the section we will prove the finite model property of logics axiomatized by NNIL-formulas as a consequence of Theorem 5.5. Our argument also uses the fact that each IPC-formula $\varphi$ can be brought into a frame-normal form of implication complexity $\leq 2$. This result seems to be more or less folklore, although a closely related form is used in [26, 19] where syntactic proofs are given. We give, instead, a semantic proof of this fact in the following.

Let us first define the frame-normal form. Given any formula $\varphi$, for each variable $p$ and constant $\perp$ occurring in $\varphi$ we let $s_{p}=p$ and $s_{\perp}=\perp$, and for each compound subformula $\psi$ of $\varphi$ we introduce a fresh variable $s_{\psi}$.

Definition 5.6. Define inductively formulas $\varphi_{+}^{\prime}$ as follows: 
- If $\varphi=p$, then define $\varphi_{+}^{\prime}=\top$.

- If $\varphi=\perp$, then define $\varphi_{+}^{\prime}=\top$.

- If $\varphi=\psi * \chi$ for $* \in\{\wedge, \vee, \rightarrow\}$, then define

$$
\varphi_{+}^{\prime}=\psi_{+}^{\prime} \wedge \chi_{+}^{\prime} \wedge\left(\left(s_{\psi} * s_{\chi}\right) \leftrightarrow s_{\varphi}\right) .
$$

Define $\varphi^{\prime}=\varphi_{+}^{\prime} \rightarrow s_{\varphi}$.

Observe that most conjuncts in $\varphi_{+}^{\prime}$ are NNIL-formulas, except for subformulas of the form $\left(s_{\psi} \rightarrow s_{\chi}\right) \rightarrow s_{\psi \rightarrow \chi}$. We now show that $\varphi$ and $\varphi^{\prime}$ are frame-equivalent to each other, and thus the formula $\varphi^{\prime}$ can be viewed as a normal form for IPC-formulas over frames.

Proposition 5.7. For any frame $\mathfrak{F}$, we have that $\mathfrak{F} \models \varphi \Longleftrightarrow \mathfrak{F} \models \varphi^{\prime}$.

Proof. To prove the proposition, we first prove the following claim.

Claim 1: For any formula $\varphi$, any model $\mathfrak{M}$ and any node $w$ in $\mathfrak{M}$, we have that $\mathfrak{M}, w \models \varphi_{+}^{\prime} \Longrightarrow \mathfrak{M}, w \models \varphi \leftrightarrow s_{\varphi}$.

Proof of Claim 1. We prove the claim by induction on $\varphi$. If $\varphi=p$ or $\perp$, then $s_{\varphi}=\varphi$ by definition, thus the claim holds trivially.

Suppose $\varphi=\psi * \chi$ for $* \in\{\wedge, \vee, \rightarrow\}$. Assume that $\mathfrak{M}, w \models \varphi_{+}^{\prime}$, i.e., $\mathfrak{M}, w \models \psi_{+}^{\prime} \wedge \chi_{+}^{\prime} \wedge\left(\left(s_{\psi} * s_{\chi}\right) \leftrightarrow s_{\varphi}\right)$. By the induction hypothesis, $\mathfrak{M}, w \models$ $\psi \leftrightarrow s_{\psi}$ and $\mathfrak{M}, w \models \chi \leftrightarrow s_{\chi}$, implying $\mathfrak{M}, w \models(\psi * \chi) \leftrightarrow\left(s_{\psi} * s_{\chi}\right)$. Since $\mathfrak{M}, w \models\left(s_{\psi} * s_{\chi}\right) \leftrightarrow s_{\varphi}$, we obtain $\mathfrak{M}, w \models(\psi * \chi) \leftrightarrow s_{\varphi}$, as required.

Now, to prove the direction " $\Longrightarrow$ " of the proposition, it suffices to prove that $\mathfrak{M}, w \models \varphi \Longrightarrow \mathfrak{M}, w \models \varphi^{\prime}$ holds for any model $\mathfrak{M}$ and any node $w$ in $\mathfrak{M}$. Suppose $\mathfrak{M}, w \models \varphi$ and $\mathfrak{M}, u \models \varphi_{+}^{\prime}$ for some successor $u$ of $w$. By Claim $1, \mathfrak{M}, u \models \varphi \leftrightarrow s_{\varphi}$, thus $\mathfrak{M}, u \models s_{\varphi}$, thereby $\mathfrak{M}, w \models \varphi^{\prime}$.

For the direction "£", suppose $(\mathfrak{F}, V), w \not \models \varphi$ for some valuation $V$ on $\mathfrak{F}$ and $w$ in $\mathfrak{F}$. Let $V^{\prime}$ be a valuation on $\mathfrak{F}$ such that $V^{\prime}\left(s_{\psi}\right)=V(\psi)$ for every subformula $\psi$ of $\varphi$.

Claim 2: $\left(\mathfrak{F}, V^{\prime}\right) \models \varphi_{+}^{\prime}$.

Proof of Claim 2. We prove the claim by induction on the subformulas $\psi$ of $\varphi$. If $\psi=p$ or $\perp$, then $\psi_{+}^{\prime}=\top$ and the claim holds trivially. Suppose $\psi=\theta * \chi$ for $* \in\{\wedge, \vee, \rightarrow\}$. Then $\psi_{+}^{\prime}=\theta_{+}^{\prime} \wedge \chi_{+}^{\prime} \wedge\left(\left(s_{\theta} * s_{\chi}\right) \leftrightarrow s_{\psi}\right)$. By the induction hypothesis, we have that $\left(\mathfrak{F}, V^{\prime}\right) \models \theta_{+}^{\prime} \wedge \chi_{+}^{\prime}$. Moreover, by the definition, $V^{\prime}\left(s_{\theta}\right)=V(\theta), V^{\prime}\left(s_{\chi}\right)=V(\chi)$ and $V(\psi)=V^{\prime}\left(s_{\psi}\right)$, which by 
a simple inductive argument imply that $V^{\prime}\left(s_{\theta} * s_{\chi}\right)=V(\theta * \chi)=V^{\prime}\left(s_{\psi}\right)$. Thus $\left(\mathfrak{F}, V^{\prime}\right) \models\left(s_{\theta} * s_{\chi}\right) \leftrightarrow s_{\psi}$.

To complete the proof we need to show that $\left(\mathfrak{F}, V^{\prime}\right) \not \varphi^{\prime}$, which can be reduced to showing that $\left(\mathfrak{F}, V^{\prime}\right), w \not \models \varphi_{+}^{\prime} \rightarrow s_{\varphi}$. By Claim 2, we have that $\left(\mathfrak{F}, V^{\prime}\right), w \models \varphi_{+}^{\prime}$. It then follows from Claim 1 that $\left(\mathfrak{F}, V^{\prime}\right), w \models \varphi \leftrightarrow s_{\varphi}$. Since $V^{\prime}$ and $V$ agree on the valuation of all propositional variables occurring in $\varphi$, the assumption $(\mathfrak{F}, V), w \not \models \varphi$ implies that $\left(\mathfrak{F}, V^{\prime}\right), w \not \models \varphi$, which gives $\left(\mathfrak{F}, V^{\prime}\right), w \not \models s_{\varphi}$, as desired.

Finally, we are in a position to prove the finite model property for logics axiomatized by NNIL- or MR-formulas.

Theorem 5.8. If $L$ is axiomatized by NNIL- or MR-formulas then $L$ has the finite model property.

Proof. Assume that $L \nvdash \varphi$ for some formula $\varphi$, and by Proposition 5.7, we may further assume that $\varphi$ is in the frame-normal form $\varphi_{+}^{\prime} \rightarrow s$ with $n$ propositional variables. Then $\varphi$ is falsified on an $n$-model $\mathfrak{M}$ on a rooted descriptive $L$-frame $\mathfrak{F}$. Let $\mathfrak{N}$ be the finite color-preserving submodel of $\mathfrak{M}$ with the same root given by Theorem 5.5. The underlying frame $\mathfrak{G}$ of $\mathfrak{N}$ is obviously a substructure of $\mathfrak{M}$, and thus $\mathfrak{G}$ is an $L$-frame by Corollary 4.13 ,

It remains to show that $\mathfrak{N}$ falsifies $\varphi=\varphi_{+}^{\prime} \rightarrow s$. Assume w.l.o.g. that the root $v$ of $\mathfrak{M}$ makes $\varphi_{+}^{\prime}$ true and $s$ false. By the construction, $v$ is also the root of $\mathfrak{N}$, and $\mathfrak{N}, v \not \models s$. It remains to prove that $\mathfrak{N}, v \models \varphi_{+}^{\prime}$. As pointed out already, most conjuncts in $\varphi_{+}^{\prime}$ are NNIL-formulas, and thus remain true in the submodel $\mathfrak{N}$. It is left to check that $v$ makes the formulas of the form $(p \rightarrow q) \rightarrow r$ true in $\mathfrak{N}$. Assuming that $w$ is a node in $\mathfrak{N}$ such that $\mathfrak{N}, w \not \neq r$, we need to show that $\mathfrak{N}, w \forall \models p \rightarrow q$. Now, since $\mathfrak{M}, w \models(p \rightarrow q) \rightarrow r$ and $\mathfrak{M}, w \not \models r$, we have $\mathfrak{M}, w \not \models p \rightarrow q$, so there must exist a successor $u$ of $w$ in $\mathfrak{M}$ such that $\mathfrak{M}, u \models p$ and $\mathfrak{M}, u \not \models q$. Since $\mathfrak{N}$ is a color-preserving submodel of $\mathfrak{M}$, there is a successor $u_{0}$ of $w$ in $\mathfrak{N}$ such that $\mathfrak{N}, u_{0} \models p$ and $\mathfrak{N}, u_{0} \not \models q$, which implies that $\mathfrak{N}, w \not \models p \rightarrow q$, as required.

\section{Open problems}

In the above we hope to have brought more clarity to the role of NNILformulas, both in models and in frames. We think this opens up a number of possibilities for future research. We enumerate some of them.

(1). In [5] the $[\wedge, \rightarrow]$-fragment of IPC was studied using finite duality for distributive lattices and universal models leading to results about how 
the universal model for that fragment fits into the overall universal model of IPC, to results about interpolation, and to the relationship of the subframe formulas connected to that fragment with the Jankov-de Jongh formulas. A similar investigation of the NNIL-fragment seems indicated, and should also throw light on the intriguing relationship between those two fragments.

(2). A clear goal for research will be a characterization of the subclass of those modal subframe logics that are closed under arbitrary substructures in the same way that all intermediate subframe logics are. Such logics obviously exist, is a syntactic characterization too much to hope for?

(3). In 29] the 2-universal model was used to initiate a study of subframe logics axiomatized by NNIL-formulas with 2 variables, for example towards the construction of characteristic frames. This study can be continued and extended to 3 variables using the 3 -universal model.

(4). In [6] ONNILLI-formulas were introduced, which are strongly related to NNIL-formulas. The universal models for NNIL-formulas may, either directly be useful for the study of ONNILLI-formulas and the stable logics they axiomatize, or indirectly, in the construction of their own universal models.

(5). Construction of the concrete 3-universal model (as far as it goes) with computer assistance may well increase insight in more-variable NNILformulas.

(6). Construction of a syntactically defined class of formulas preserved under cofinal submodels extending NNIL and study of its properties, and construction of universal models. Presumably such a class should contain the cofinal subframe formulas of [3, 4].

(7). It seems worthwhile to investigate the concept of universal model in general for locally finite fragments of IPC and other logics.

\section{Funding}

The third author was supported by grants 308712 and 330525 of the Academy of Finland, and Research Funds of the University of Helsinki.

\section{Acknowledgments}

The authors would like to thank Nick Bezhanishvili for useful discussions on parts of the paper, and two anonymous referees for valuable suggestions. They also thank Silvio Ghilardi for showing them the connection to the concept of relatively open, which was also pointed out by one of the referees. 


\section{References}

[1] F. Bellissima. Finitely generated free Heyting algebras. Journal of Symbolic Logic, 51:152-165, 1986.

[2] G. Bezhanishvili and S. Ghilardi. An algebraic approach to subframe logics. Intuitionistic case. Ann. Pure Appl. Logic, 147(1-2):84-100, 2007.

[3] N. Bezhanishvili. Lattices of Intermediate and Cylindric Modal Logics. PhD thesis, University of Amsterdam, 2006. Available at http://www.illc.uva.nl/Research/Publications/Dissertations/DS-2006-02.text.pdf.

[4] N. Bezhanishvili. Frame based formulas for intermediate logics. Studia Logica, 90:139-159, 2008.

[5] N. Bezhanishvili, D. Coumans, S. van Gool, and D. de Jongh. Duality and universal models for the meet-implication fragment of IPC. In M. Aher, D. Hole, E. Jeřábek, and C. Kupke, editors, Logic, Language, and Computation: 10th International Tbilisi Symposium on Logic, Language, and Computation, TbiLLC 2013, Gudauri, Georgia, September 23-27, 2013: revised selected papers, volume 8984 of Lecture Notes in Computer Science, pages 97-116. Springer-Verlag, 2015.

[6] N. Bezhanishvili and D. de Jongh. Stable logics in intuitionistic logic. Notre Dame Journal of Formal Logic, 59(3):307-324, 2018.

[7] N. Bezhanishvili, D. de Jongh, A. Tzimoulis, and Z. Zhao. Universal models for the positive fragment of intuitionistic logic. In Logic, Language, and Computation - 11th International Tbilisi Symposium, TbiLLC 2015, Tbilisi, Georgia, September 21-26, 2015, Revised Selected Papers, pages 229-250, 2015.

[8] A. Chagrov and M. Zakharyaschev. Modal logic, volume 35 of Oxford Logic Guides. The Clarendon Press, New York, 1997.

[9] C. Contrini and Y. Gurevich. Transitive infon logics. Rev. Symb. Log., 6(2):281-304, 2013.

[10] D. van Dalen. Intuitionistic Logic. In D. Gabbay and F. Guenthner, editors, Handbook of Philosophical Logic, volume 3, pages 225-339. Kluwer, Reidel, Dordrecht, 1986. 
[11] D. de Jongh. Investigations on the Intuitionistic Propositional Calculus. PhD thesis, University of Wisconsin, 1968. Available at http://www.illc.uva.nl/Research/Publications/Dissertations/HDS-05-Dick-de-Jongh .

[12] D. de Jongh and A. Visser. Embeddings of Heyting Algebras. In Wilfrid Hodges, Martin Hyland, Charles Steinhorn, and John Truss, editors, Logic: from foundations to applications, European logic coll. 1993, Oxford Science Publications, pages 187-213. Clarendon Press, Oxford, 1996.

[13] K. Fine. Logics containing K4. II. J. Symbolic Logic, 50(3):619-651, 1985.

[14] S. Ghilardi. Free heyting algebras as bi-heyting algebras. Math. Rep. Acad. Sci. Canada XVI., 6:240-244, 1992.

[15] R. Grigolia. Free Algebras of Non-Classical Logics. "Metsniereba", Tbilisi, 1987. (Russian).

[16] A. Hendriks. Computations in Propositional Logic. PhD thesis, University of Amsterdam, $1996 . \quad$ Available at http://www.illc.uva.nl/Research/Publications/Dissertations/DS-1996-01.text.ps.gz.

[17] J. Ilin. Filtration revisited: lattices of stable non-classical logics. $\mathrm{PhD}$ thesis, University of Amsterdam, 2018.

[18] J. Ilin, D. de Jongh, and F. Yang. NNIL axioms have the finite model property. In J. van Eijck, R. Iemhoff, and J. Joosten, editors, Liber Amicorum Alberti, volume 30 of Tributes, pages 177-185. College publications, 2016.

[19] E. Jerabek. A note on the substructural hierarchy. Mathematical Logic Quarterly, 62(1-2):102-110, 2016.

[20] D. de Jongh and F. Yang. Jankov's theorems for intermediate logics in the setting of universal models. In N. Bezhanishvili et al., editor, Proceedings of TbiLLC'09: International Conference on Logic, Language and Computation, volume 6618 of LNAI, pages 53-76. Springer-Verlag, 2011.

[21] I. Nishimura. On formulas of one variable in intuitionistic propositional calculus. Journal of Symbolic Logic, 25:327-331, 1960. 
[22] G.R. Renardel de Lavalette, A. Hendriks, and D. de Jongh. Intuitionistic Implication without Disjunction. J. Log. Comp., 22(3):375-404, 2012 .

[23] L. Rieger. On the lattice theory of Brouwerian propositional logic. Acta fac. rerum nat. Univ. Car., 189:1-40, 1949.

[24] V. V. Rybakov. Admissibility of Logical Inference Rules. Elsevier, 1997.

[25] V.B. Shehtman. Rieger-Nishimura lattices. Soviet Mathematics Doklady, 19:1014-1018, 1978.

[26] R. Statman. Intuitionistic propositional logic is polynomial-space complete. Theor. Comp. Sc., 9:67-72, 1979.

[27] A. Visser. Evaluation, provably deductive equivalence in Heyting's Arithmetic. Technical Report 4, Dept. of Philosophy, Utrecht University, 1985.

[28] A. Visser, D. de Jongh, J. van Benthem, and G. Renardel de Lavalette. NNIL a study in intuitionistic logic. In A. Ponse, M. de Rijke, and Y. Venema, editors, Modal logics and Process Algebra: a bisimulation perspective, pages 289-326, 1995.

[29] F. Yang. Intuitionistic subframe formulas, NNIL-formulas and nuniversal models. Master's Thesis, MoL-2008-12, ILLC, University of Amsterdam, 2008.

[30] M. Zakharyaschev. Syntax and semantics of superintuitionistic logics. Algebra and Logic, 28(4):262-282, 1989. 